\title{
Latent Membrane Protein 1 Promotes Tumorigenesis Through Upregulation of PGC1 $\beta$ Signaling Pathway
}

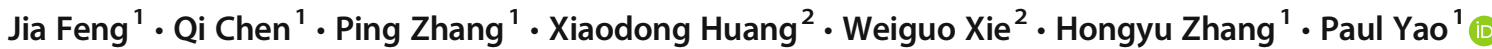

Accepted: 22 December 2020 / Published online: 9 January 2021

(C) The Author(s) 2021

\begin{abstract}
Natural killer/T-cell lymphoma (NKTCL) is an aggressive Epstein-Barr virus (EBV)-associated non-Hodgkin lymphoma with poor prognosis. In this study, we aimed to investigate the potential mechanism of latent membrane protein 1 (LMP1)-mediated tumorigenesis and provide a novel therapeutic strategy for targeting the EBV DNA genome. We found that LMP1 upregulated the expression of peroxisome proliferator-activated receptor- $\gamma(\operatorname{PPAR} \gamma)$ coactivator- $1 \beta$ (PGC1 $\beta$ ) through activation of nuclear factor- $\mathrm{KB}(\mathrm{NF}-\mathrm{kB})$. Furthermore, the activated PGC1 $\beta$ upregulated the expression of 8-oxoguanine DNA glycosylase (OGG1) through the coactivation of nuclear respiratory factor 1 (NRF1) and GA-binding protein $\alpha(\mathrm{GABP} \alpha)$, preventing reactive oxygen species (ROS)-mediated base incision in the EBV genome and favoring its survival. Interruption of hexokinase domain component 1 (HKDC1) by either shRNA or Tf-D-HKC8 peptide suppressed the interaction of HKDC1 with voltage-dependent anion channel 1 (VDAC1), triggering mitochondrial dysfunction and excessive generation of ROS, thus resulting in EBV suppression through ROS-mediated DNA damage. Suppression of the EBV genome inhibited the expression of the LMP1/PGC1 $\beta / \mathrm{HKDC} 1 /$ OGG1 signaling pathway, forming a positive feed forward loop for the generation of ROS, hence inhibiting the EBV genome and subsequent EBV-associated tumor development. We concluded that LMP1 triggers EBV-associated tumorigenesis through activation of the PGC1 $\beta$ pathway. This study provided a novel therapeutic strategy for the treatment of EBV-associated tumors by targeting HKDC1.
\end{abstract}

Keywords Hexokinase domain containing $1 \cdot$ Latent membrane protein $1 \cdot$ Nuclear factor- $\mathrm{kB} \cdot 8$-oxoguanine DNA glycosylase $\cdot$ Peroxisome proliferator-activated receptor- $\gamma$ coactivator $1 \beta$

\section{Introduction}

Natural killer/T-cell lymphoma (NKTCL) is an aggressive Epstein-Barr virus (EBV)-associated non-Hodgkin lymphoma with a high prevalence in most Asian populations [1-3]. Multiple pathogenic pathways have been reported to be involved in NKTCL [4]. Many different therapeutic targets, including programmed cell death 1 (PD-1) and Pglycoprotein (P-gp), have been extensively investigated and

Jia Feng and Qi Chen contributed equally to this work.

Hongyu Zhang

zyiqu@163.com

Paul Yao

vasilis112@yahoo.com

1 Department of Hematology, Peking University Shenzhen Hospital, 518036 Shenzhen, People's Republic of China

2 Institute of Rehabilitation Center, Tongren Hospital of Wuhan University, 430060 Wuhan, People's Republic of China their targeting was shown to result in significant improvements in symptoms [5-7].Whereas, many cases were demonstrated to eventually relapse, resulting in poor prognosis $[8,9]$. Many studies have shown that infection with EBV might be a potential driving force for tumor development, such as through oxidative stress-mediated EBV DNA damage, thus indicating EBV DNA as a novel therapeutic target in EBVassociated tumors $[10,11]$.

The latent membrane protein 1 (LMP1) encoded by EBV is known to be a direct target gene of Epstein-Barr virus nuclear antigen 2 (EBNA2). As an oncogene [12], LMP1 has been reported to act as a master metabolic regulator in many EBVassociated tumor cells by potentiating aerobic glycolysis, a condition known as the Warburg effect [13]. Briefly, LMP1 has 6 hydrophobic membrane-spanning domains and a 200amino acid cytoplasmic carboxyl terminus, which is comprised of 2 domains, named transformation effector sites 1 and 2 (TES1 and TES2), respectively. These 2 domains are known to be responsible for the transformation of B-cells and the activation of the nuclear factor- $\mathrm{KB}(\mathrm{NF}-\mathrm{kB})$ [14], which 
then translocates into the nucleus to activate its target genes [15]. It has been reported that activated NF-KB prevents cell apoptosis [16] and favors tumorigenesis. However, the detailed mechanisms underlying this effect remain largely unknown [17].

The peroxisome proliferator-activated receptor- $\gamma(\operatorname{PPAR} \gamma)$ coactivator-1 (PGC1) family, which includes members such as PGC1 $\alpha$ and PGC1 $\beta$ [18], has been reported to play an important role in maintaining the energy balance through the metabolism of glucose and lipids. More specifically, PGC1 $\alpha$ and $\mathrm{PGC} 1 \beta$ have been shown to function as transcriptional coactivators regulating many downstream target genes [19]. It has been recently reported that PGC1 $\beta$ is critical in tumorigenesis through the regulation of mitochondrial biogenesis, glycolysis metabolism [20-23], and oxidative stress [24]. We recently found that $\mathrm{PGC} 1 \beta$ regulates tumor growth and metastasis through a lactate dehydrogenase A (LDHA)-mediated glycolytic metabolism [25], as well as through the expression of SREBP 1-mediated hexokinase domain component 1 (HKDC1) [26]. Hexokinase (HK) is a rate-limiting enzyme known to phosphorylate hexose sugars to maintain the glucose metabolism [27]. Additionally, it has been shown to interact with the voltage-dependent anion channel (VDAC) protein, thus regulating the mitochondrial membrane potential (MMP) and apoptosis [28]. It has also been reported to be involved in tumor initiation and maintenance [29]. Similarly, HKDC1, another novel HK isoform, has been recently found to play a potential role in tumorigenesis through glucose homeostasis and oxidative stress [26, 30-33]. In addition, we recently found that HKDC1 C-terminal based peptides inhibited NKTCL by modulating the mitochondrial function and suppressing EBV [34]; however, the detailed mechanism remains unclear.

In this study, we aimed to investigate the potential mechanism of LMP1 protein-mediated tumorigenesis and provide a novel therapeutic strategy for targeting EBV DNA in NKTCL cells. We found that the expression of $\mathrm{PGC} 1 \beta$ was upregulated by the LMP1 protein through the activation of NF- $\mathrm{KB}$ on the PGC1 $\beta$ promoter. Furthermore, PGC1 $\beta$ was demonstrated to upregulate the expression of 8-oxoguanine DNA glycosylase (OGG1), an enzyme involved in base excision repair following DNA damage [35], through the coactivation of nuclear respiratory factor 1 (NRF1) and GA binding protein $\alpha(\mathrm{GABP} \alpha)$ binding elements on the OGG1 promoter, thus preventing EBV from oxidative stress-mediated DNA damage and favoring EBV survival. We also found that interruption of HKDC1 through either gene knockdown or HKDC1 Cterminal based peptides [36] inhibited its ability to bind with VDAC1, triggering mitochondrial dysfunction and the generation of reactive oxygen species (ROS), resulting in EBV DNA damage. This further triggered the suppression of the LMP1/PGC1 $\beta / H K D C 1 / O G G 1$ signaling pathway, providing a positive feedforward loop for the generation of ROS and suppression of the EBV DNA and EBV-mediated tumorigenesis in NKTCL cells. We concluded that the expression of LMP1 promoted tumorigenesis through the upregulation of the PGC1 $1 / \mathrm{HKDC} 1 / \mathrm{OGG} 1$ signaling pathway. Thus, the interruption of HKDC1 might be a potential novel therapeutic strategy for the treatment of EBV-associated tumors.

\section{Results}

\section{Expression of EBV LMP1 Protein Regulated the PGC1ß/OGG1 Signaling Pathway and NF-KB Activity in NKTCL Cells}

We first evaluated the gene expression of the PGC1 $\beta /$ OGG1 signaling pathway in different NKTCL cell lines, including PBMC, HANK1, NK92, SNT8, and SNK6 cells. Our results showed that the LMP1 mRNA was not expressed in healthy PBMCs, whereas significant levels of expression were observed in other NKTCL cell lines. Additionally, we found that the mRNA levels of PGC1 $\beta$ and OGG1 were increased to $178 \%$ and $164 \%$ in HANK1 cells compared with PMBC cells. We noted that other NKTCL cell lines had significantly increased expression levels similar to those observed in HANK1 cells; however, the expression levels of the NF-kB subunit p65 and GABP $\alpha$ did not show a significant difference (Fig. 1a). We also evaluated the gene expression of healthy Natural killer (NK) and PBMC cells for those genes, and the results showed no significant difference (Fig. S1). We then measured the protein levels for those genes, and detected an expression pattern similar to that of the mRNA levels (Fig. 1b and c, S2a). Next, we evaluated the potential effect of a LMP1 knockdown (shLMP1) in NKTCL cells. Our results showed that the levels of the LMP1 protein were decreased to $23 \%$ and $19 \%$ in HANK 1 and SNK6 cells, respectively. The expression of PGC1 $\beta$ was also shown to be decreased to $34 \%$ and $46 \%$, while the expression of OGG1 was decreased to $47 \%$ and $54 \%$, respectively, compared with that in the HANK1/CTL group. Whereas the expression of the NF-kB subunit $\mathrm{p} 65$ and GABP $\alpha$ showed no difference (Fig. $1 \mathrm{~d}$ and e, S2b). We also measured the activity of NF-kB p65. Our results showed that following a LMP1 knockdown in HANK1 and SNK6 cells, the activity of NF- $\mathrm{KB}$ p 65 was decreased to $35 \%$ and $39 \%$, respectively, compared with the HANK1/CTL group (Fig. 1f). Finally, we evaluated the potential effect of the expression of LMP1 in healthy PBMCs and found that following infection with a LMP1-expressing lentivirus the LMP1 protein was significantly expressed in 2 lines of PBMCs. In addition, the expression of PGC1 $\beta$ was demonstrated to be increased to $226 \%$ and $198 \%$, while the expression of OGG1 was increased to $178 \%$ and $213 \%$, compared with the PBMC1/CTL group. Again, we noted that the expression of the NF-KB subunit $\mathrm{p} 65$ and $\mathrm{GABP} \alpha$ showed no difference 
(Fig. 1g, h, S2c). We also measured the activity of NF-kB p65 following the expression of LMP1 in PBMC1 and PBMC2 cells, and found that it was increased to $235 \%$ and $263 \%$, respectively, compared with the PBMC1/CTL group (Fig. 1i). Our results suggested that the expression of PGC1 $\beta$ and OGG1 in addition to the activity of NF- $k B$ were regulated by the expression of LMP1 in NKTCL cells.

\section{Expression of PGC1 $\beta$ was Regulated By LMP1- mediated Activation of NF-KB on the PGC1 $\beta$ Promoter}

We investigated the possible molecular mechanism underlying the LMP1-mediated PGC1 $\beta$ activation. We initially generated a series of progressive $5^{\prime}$-promoter deletion constructs for the PGC1 $\beta$ promoter, and transfected them into conditional immortalized PBMCs infected with either LMP1 or empty control lentivirus for the analysis of the activity of the PGC1 $\beta$ reporter. Our results showed that the LMP1-induced activation of the PGC1 $\beta$ reporter was not altered among the -2000 , $-1600,-1200,-800,-700$, and -600 deletion constructs (numbered according to Ensembl gene ID: PPARGC1B-201 ENST00000309241.9; transcription start site was marked as 0 ), whereas the LMP1-induced activation was shown to disappear in the -500 deletion construct and subsequent deletion reporter constructs. These observations indicated that the LMP1-responsive transcriptional element was located in the range of -600 to approximately -500 on the $\mathrm{PGC} 1 \beta$ promoter (Fig. 2a). Analysis of the transcription factor databases revealed the existence of many potential binding motifs, including those for $\mathrm{AP} 1, \mathrm{Sp} 1, \mathrm{Oct} 1, \mathrm{C} / \mathrm{EBP} \varepsilon, \mathrm{NF} 1$, and $\mathrm{NF}-\mathrm{KB}$ (marked in red) located in the range of -600 to -500 on the PGC1 $\beta$ promoter (Fig. $2 b$ ). We then mutated all of these potential binding motifs on the full length $\mathrm{PGC} 1 \beta$ reporter construct (pPGC1 $\beta-2000$ ), and found that the LMP1induced activation of the reporter disappeared only in the case of the NF- $\mathrm{KB}$ mutation construct (from ggggtttcacc to ggtattcaaa, located at -529 , marked in green, Fig. 2b). This finding indicated that LMP1 induced the activation of PGC1 $\beta$ through the NF- $\mathrm{KB}$ binding motif on the PGC1 $\beta$ promoter (Fig. 2c). We then conducted ChIP analysis using antibodies for potential transcription factors located in the range of -600 to -500 . We first infected 2 PBMC lines with a LMP1expressing lentivirus and observed that only NF- $\mathrm{kB}$ p65 exhibited an increased binding ability (Fig. 2d). Furthermore, we knocked-down LMP1 in 2 NKTCL cell lines, HANK1 and SNK6, using shLMP1, and found that among all transcription factors only NF-kB p 65 had a decreased binding ability on the PGC1 $\beta$ promoter (Fig. 2e). We also evaluated the potential effects of knocking-down NF- $\mathrm{kB}$. We accordingly observed that the PGC1 $\beta$ luciferase reporter activity was significantly decreased in both HANK1 and SNK6 cells transected with either siNF-kB-p65 or siNF-kB-p50 (Fig. 2f). We also evaluated the gene expression in these constructs. Our results showed that the levels of expression of both mRNA and protein of NF-kB-p65 and NF-kB-p50 were decreased to approximately 50\% in SNK6 cells following transfection with these siRNAs, indicating successful gene knockdown. Furthermore, the levels of expression of both PGC1 $\beta$ mRNA (Fig. $2 \mathrm{~g}$ ) and protein (Fig. $2 \mathrm{~h}$ and i, and FS3) were demonstrated to exhibit

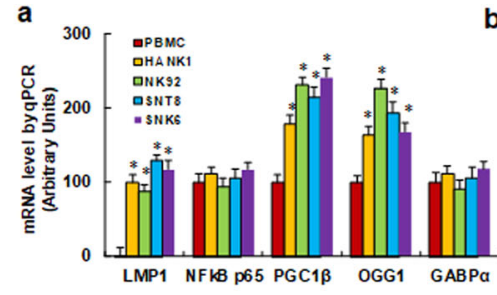

e
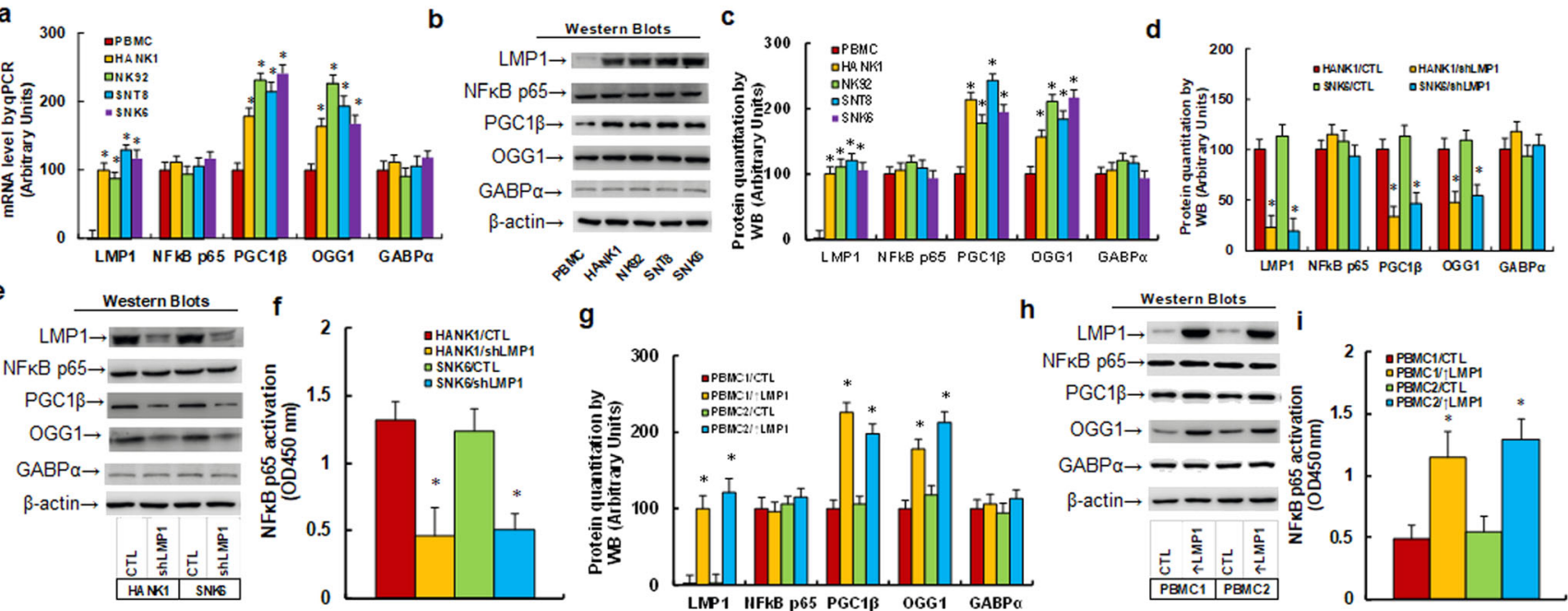

Fig. 1 Expression of EBV LMP1 protein regulates the PGC1ß/OGG1 signaling pathway and NF- $\mathrm{KB}$ activity in NKTCL cells. a Different NKTCL cell lines, including PBMC, HANK1, NK92, SNT8 and SNK6 cells, were used for mRNA analysis, $\mathrm{n}=4$. $*, P<0.05$, vs. PBMC group. b Representative pictures for Western Blotting. $\mathbf{c}$ Protein quantitation for (b), $\mathrm{n}=5$. *, $P<0.05$, vs. PBMC group. d-f The LMP1 was knockdown by lentivirus in HANK1 and SNK6 cells for analysis. d Protein quantitation for Western Blotting, $\mathrm{n}=5$; e Representative pictures for (d); f NF-kB p65 activation assay, $\mathrm{n}=5$; *, $P<0.05$, vs. HANK1/CTL. g-i The LMP1 was overexpressed by lentivirus in PBMCs for analysis. g Protein quantitation for Western Blotting, $\mathrm{n}=5$; $\mathbf{h}$ Representative pictures for (g); i NF-KB p65 activation assay, $\mathrm{n}=5$; *, $P<0.05$, vs. $\mathrm{PBMC} 1 / \mathrm{CTL}$. Data were expressed as mean $\pm \mathrm{SEM}$ 

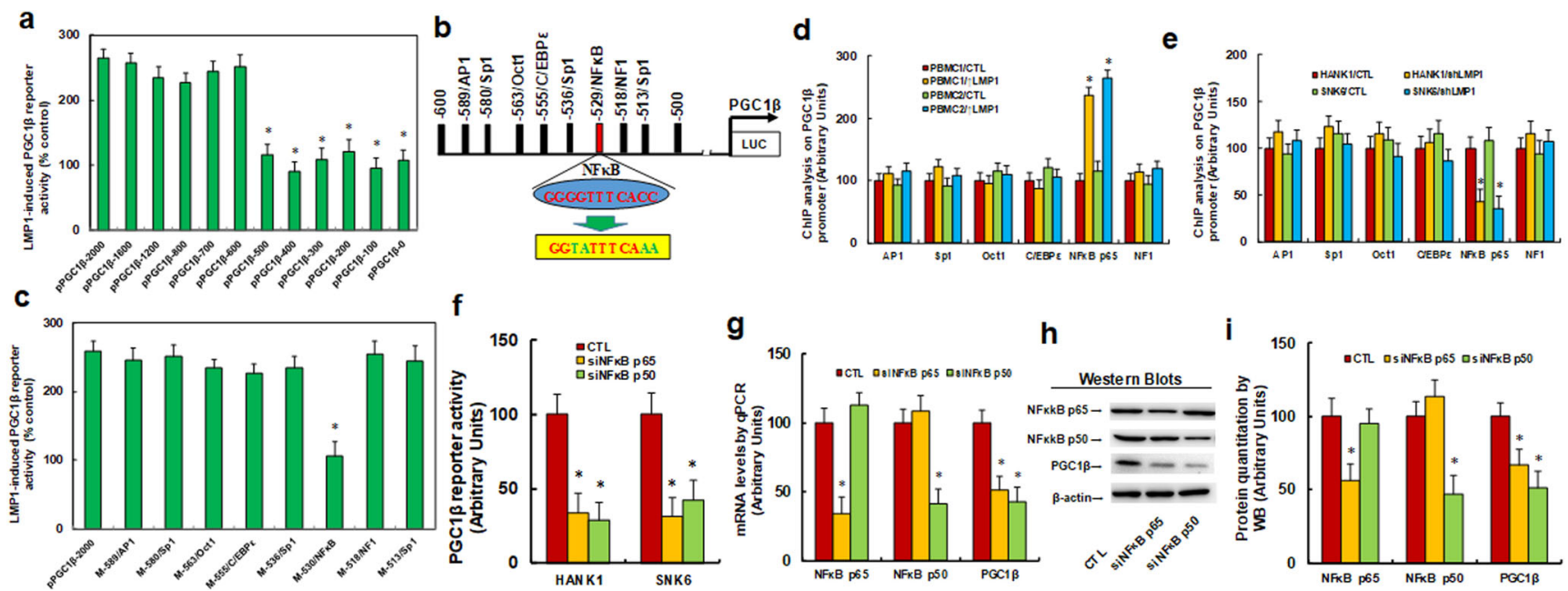

Fig. 2 PGC1 $\beta$ expression is regulated by LMP1-mediated NF- $\mathrm{KB}$ activation on the $\mathrm{PGC} 1 \beta$ promoter. a The conditionally immortalized PBMCs were infected by either LMP1 or empty control (CTL) lentivirus for 2 days, and then the cells were transiently transfected by either PGC1 $\beta$ full length (pPGC1 $\beta-2000$ ) or deletion reporter plasmids. After 24 hours, the LMP1-induced PGC1 $\beta$ reporter activities were calculated as the relative percentage ( $\%$ control) in comparison to lentivirus empty control (CTL) infected cells. *, $P<0.05$, vs. pPGC1 $\beta-2000$ group, $\mathrm{n}=5$. b The schematic picture for the potential transcriptional binding motif in the range of $-600 \sim 500$ (from transcription start site) on the PGC1 $\beta$ promoter in addition to the potential NF-KB binding site are marked in red, and the related mutation sites are marked in green. $\mathbf{c}$ The cells were transiently transfected by either the PGC1 $\beta$ full length (pPGC1 $1 \beta-2000$ )

or the specific transcriptional binding motif mutation reporter plasmids, and then the reporter activities were measured after 24 hours. *, $P<0.05$, vs. pPGC1 $\beta-2000$ group, $n=5$. $\mathbf{d}$ Immortalized PBMCs were infected by LMP1 lentivirus and then cells were used for ChIP analysis using different antibodies, and the $\mathrm{PGC} 1 \beta$ promoter was amplified and measured by qPCR, $\mathrm{n}=5$. * $P<0.05$, vs. PBMC1/CTL group. e LMP1 was knockdown by lentivirus in HANK1 and SNK6 cells for ChIP analysis on PGC1 $\beta$ promoter, $\mathrm{n}=5$. ${ }^{*}, P<0.05$, vs. HANK1/CTL group. $\mathbf{f}-\mathbf{i}$ The siRNA was used to knockdown either NF-kB p65 or p50 in HANK1 and SNK6 cells for analysis; $\mathbf{f}$ PGC1 $\beta$ reporter activity; $\mathbf{g}$ mRNA levels by qPCR; $\mathbf{h}$ Representative pictures for Western Blotting, $\mathrm{n}=5$; $\mathbf{i}$ Protein quantitation for $(\mathbf{h}) . \mathrm{n}=5$. $*, P<0.05$, vs. CTL group. Data were expressed as mean \pm SEM

significant decreases as a result of the siRNA treatment for both p65 and p50 subunits. Our results indicated that the expression of PGC1 1 was regulated by the LMP1-mediated activation of NF- $\mathrm{KB}$ on the $\mathrm{PGC} 1 \beta$ promoter.

\section{Expression of OGG1 was Regulated By PGC1 $\beta$ - mediated Activation of NRF1/GABPa on the OGG1 Promoter}

We also investigated the potential mechanism of the PGC1 $\beta$-mediated activation of the expression of OGG1. We generated a series of progressive 5'-promoter deletion constructs for the OGG1 promoter, and transfected these constructs into conditional immortalized PBMCs infected with either PGC1 $\beta$ or empty control lentivirus for the analysis of the activity of the OGG1 reporter. We respectively found that the PGC1 $\beta$-induced activation of the OGG1 reporter was not altered among the $-2000,-1600,-1200$, $-800,-600$, and -400 , and -300 deletion constructs (numbered according to the Ensembl gene ID: OGG1-201 ENST00000302003.11; transcription start site was marked as 0 ), whereas the PGC1 $\beta$-induced activation was demonstrated to be partly decreased in the -200 and -150 deletion constructs, and completely disappeared in the -50 and 0 deletion constructs. These findings indicated that the

PGC1 $1 \beta$-responsive transcriptional element was located in the range of -300 to approximately -50 on the OGG1 promoter (Fig. 3a). Moreover, analysis of transcription factor databases revealed the presence of many potential binding motifs, including the $\mathrm{Sp} 1, \mathrm{GABP} \alpha$ (marked in red), RXR $\beta, A P 2 \alpha, N F 1$, and NRF1 (marked in red) binding sites located in the range of -300 to -50 on the OGG1 promoter (Fig. 3b). We then mutated all of these potential binding motifs on the full length OGG1 reporter construct (pOGG1-2000) and found that the PGC1 $\beta 1$-induced activation of the reporter was significantly decreased in both GABP $\alpha$ (from ggggaagg to ggttccgg, in green) and NRF1 (from cgcgcgagcgcct to cgtatagatatact, in green) mutation constructs (Fig. 3b). This finding indicated that PGC1 $\beta$ induced the activation of OGG1 through GABP $\alpha$ (located at -242) and NRF1 (located at -105) binding motifs on the OGG1 promoter (Fig. 3c). We then generated single mutations, as well as a double mutation on the GABP $\alpha$ and NRF1 binding sites in the pOGG1 fulllength construct (M-242/GABP $\alpha-105 / N R F 1)$ and found that whereas each single mutant led to the partial decrease in the activation of OGG1, the double mutant completely diminished the PGC1 $\beta$-induced activation of OGG1 (Fig. 3d). We then conducted ChIP analysis using antibodies for potential transcription factors located in the range of 


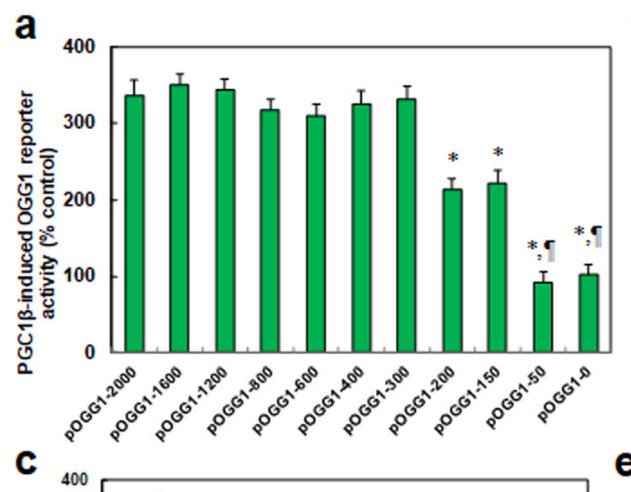

b

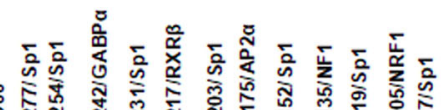

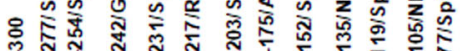

ii i i i i i i

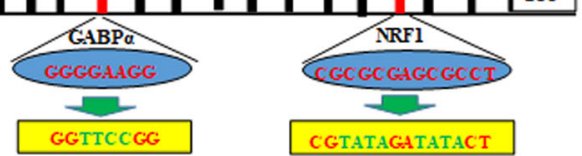

d

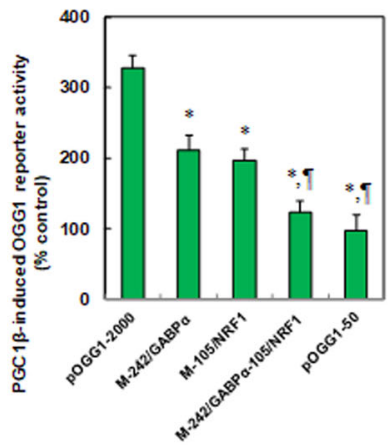

c

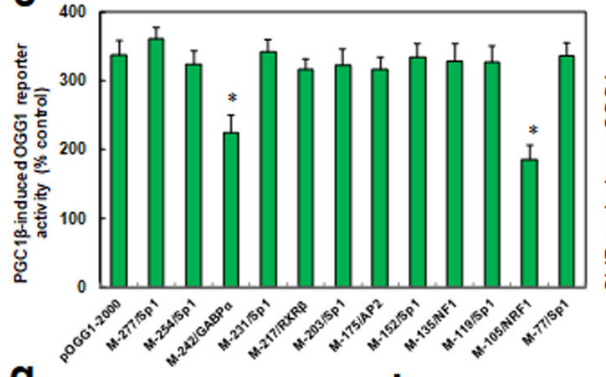

e ${ }_{300}$

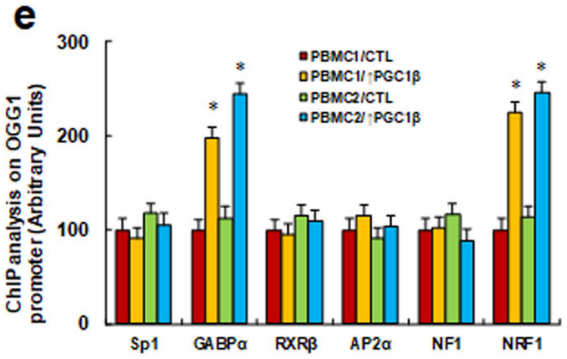

g
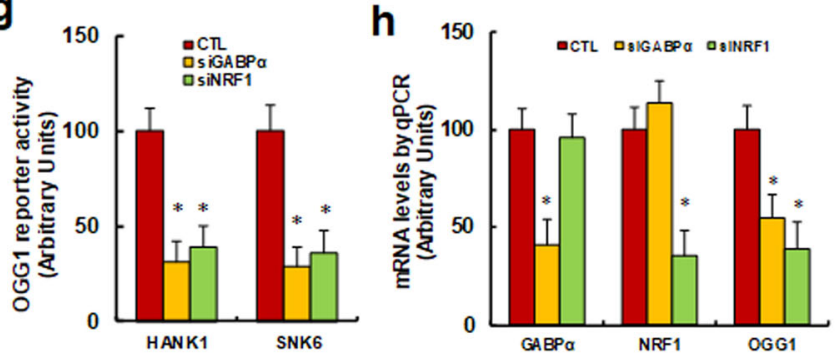

Fig. 3 OGG1 expression is regulated by PGC1 $\beta$-mediated NRF1/ GABP $\alpha$ activation on the OGG1 promoter. a The conditionally immortalized PBMCs were infected by either PGC1 $\beta$ or empty control (CTL) lentivirus for 2 days, and then the cells were transiently transfected by either OGG1 full length (pOGG1-2000) or deletion reporter plasmids. After 24 hours, the PGC1 $\beta$-induced OGG1 reporter activities were calculated as the relative percentage (\% control) in comparison to lentivirus empty control (CTL) infected cells. *, $P<0.05$, vs. pOGG1-2000 group, $\mathrm{n}=5$. $\mathbf{b}$ The schematic picture for the potential transcriptional binding motif in the range of -300 -50 (from transcription start site) on the OGG1 promoter; the potential binding sites for NRF1 and GABP $\alpha$ are marked in red, and the related mutation sites are marked in green. $\mathbf{c}$ The cells were transiently transfected by either OGG1 full length (pOGG1-2000) or the specific transcriptional binding motif mutation reporter plasmids, and then the reporter activities were measured after 24 hours. ${ }^{*}, P<0.05$,

-300 to -50 . To this end, we first infected 2 PBMC lines with a PGC1-expressing lentivirus and observed that both GABP $\alpha$ and NRF1 exhibited an increased binding ability (Fig. 3e). Furthermore, we knocked-down PGC1 $\beta$ in 2 NKTCL cell lines, HANK1 and SNK6, using shPGC1 $\beta$, and respectively found that both GABP $\alpha$ and NRF1 exhibited decreased binding ability on the OGG1 promoter (Fig. 3f). We also evaluated the potential effect of knocking-down either the GABP $\alpha$ or NRF1 gene. We noted that the OGG1 luciferase reporter activity was significantly decreased as a result of treatment of both HANK1 and SNK6 cells with either siGABP $\alpha$ or siNRF1 (Fig. 3g). i

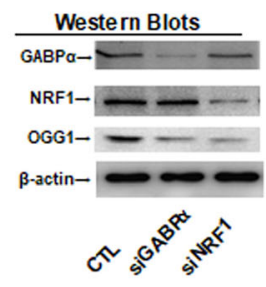

f

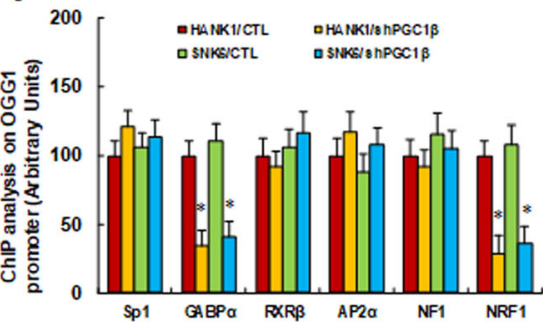

j

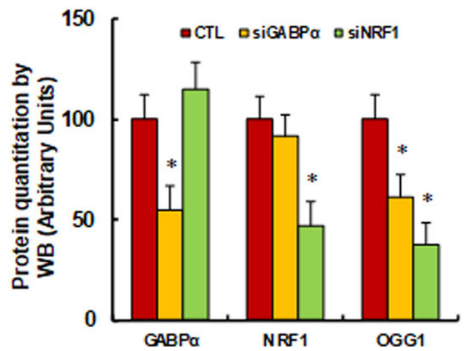

vs. pOGG1-2000 group, $n=5$. d The cells were transiently transfected by either OGG1 full length (pOGG1-2000), single mutant, or double mutants for NRF1 and $\mathrm{GABP} \alpha$, and then the reporter activities were measured after 24 hours. ${ }^{*}, P<0.05$, vs. pOGG1-2000 group, $\mathrm{n}=5$. e Immortalized PBMCs were infected by PGC1 $\beta$ lentivirus; then, cells were used for ChIP analysis using different antibodies, and the PGC1 $\beta$ promoter was amplified and measured by $\mathrm{qPCR}, \mathrm{n}=5$. ${ }^{*}, P<0.05$, vs. PBMC1/CTL group. $\mathbf{f}$ PGC1 $\beta$ was knockdown by lentivirus in HANK1 and SNK6 cells for ChIP analysis on PGC1 $\beta$ promoter, $\mathrm{n}=5$. *, $P<0.05$, vs. HANK1/CTL group. g-j The siRNA was used to knockdown either GABP $\alpha$ or NRF1 in HANK1 and SNK6 cells for analysis; $\mathbf{g}$ OGG1 reporter activity; $\mathbf{h}$ mRNA levels by qPCR; i Representative pictures for Western Blotting, $\mathrm{n}=5$; $\mathbf{j}$ Protein quantitation for $(\mathbf{i}) . \mathrm{n}=5$. *, $P<0.05$, vs. CTL group. Data were expressed as mean \pm SEM

We also evaluated the levels of gene expression following treatment with these siRNAs and noted that the levels of both the mRNA and protein expression of both GABP $\alpha$ and NRF1 were significantly decreased in SNK6 cells, indicating successful gene knockdown. Furthermore, we observed that the levels of expression of both OGG1 mRNA (Fig. 3h) and protein (Fig. 3i and j and FS4) exhibited a significant decrease as a result of the siRNA treatment for both GABP $\alpha$ and NRF1. Our results thus indicated that the expression of OGG1 was regulated by the PGC1 $\beta$ mediated activation of either or both GABP $\alpha$ and NRF1 on the PGC1 $\beta$ promoter. 


\section{Tf-D-HKC8 Peptide-mediated Interruption of HKDC1 Induced Suppression of PGC1B/OGG1 and Mitochondrial Dysfunction}

We evaluated the potential effect of the Tf-D-HKC8 peptide on the PGC1 $\beta /$ OGG1 signaling pathway and mitochondrial function in SNK6 cells. We first evaluated the gene expression of the PGC1 $\beta /$ OGG1 signaling pathway. Our results showed that treatment with Tf-D-HKC8 (Tf-D-HKC8/CTL) significantly decreased the mRNA levels of PGC1 $\beta, \mathrm{HDKC} 1$, and OGG1. Conversely, infection with a SOD2-expressing lentivirus (Tf-D-HKC8/ $\uparrow$ SOD2) completely reversed this effect, whereas infection with an OGG1-expressing lentivirus (Tf-D-HKC8/^OGG1) had little effect, compared with the control group (Tf-D-Scram/CTL). In addition, we observed that the VDAC1 mRNA levels did not change as a result of any of these treatments, while overexpression of SOD2 and OGG1 was shown to significantly increase each of the related mRNA levels, indicating successful infection (Fig. 4a). We then measured the protein levels for those genes, and found an expression pattern similar to that of the mRNA levels (Fig. 4b and c, S5a). Next, we evaluated the interaction between VDAC1 and HKDC1 using immunoprecipitation (IP)/ western blotting (WB) techniques. Our results showed that treatment with Tf-D-HKC8/CTL significantly interrupted the binding ability of HKDC1 with VDAC1, reducing it to $36 \%$ compared with the Tf-D-Scram/CTL group, whereas overexpression of either SOD2 or OGG1 had no effect (Fig. 4d and e, $\mathrm{S} 5 \mathrm{~b})$. We then evaluated the effect of the Tf-D-HKC8 peptide on reporter activities and found that treatment with Tf-DHKC8/CTL significantly decreased the luciferase reporter activities of both PGC1 $\beta$ and OGG1, with overexpression of SOD2 completely reversing this effect, whereas overexpression of OGG1 had no effect (Fig. 4f). We also evaluated the effect of the Tf-D-HKC8 peptide on the binding ability of transcription factors on promoters. Our results showed that treatment with Tf-D-HKC8/CTL significantly decreased the ability of PGC1 $\beta$ and SREBP1 to bind to the HKDC1 promoter, whereas we did not observe any effect on the binding ability of PGC1 $\alpha$ (Fig. 4g). Additionally, treatment with TfD-HKC8/CTL was demonstrated to significantly decrease the ability of PGC1 $1 \beta, \operatorname{GABP} \alpha$, and NRF1 to bind to the OGG1 promoter (Fig. 4h). Again, we noted that overexpression of SOD2 completely reversed this effect, whereas overexpression of OGG1 had no effect. We also evaluated the changes in the activity of hexokinase as a result of different treatments. We accordingly found that treatment with Tf-D-HKC $8 / C T L$ increased the levels of the activity of hexokinase to $146 \%$ compared with the Tf-D-Scram/CTL group, whereas little effect was noted following infection with either SOD2 or
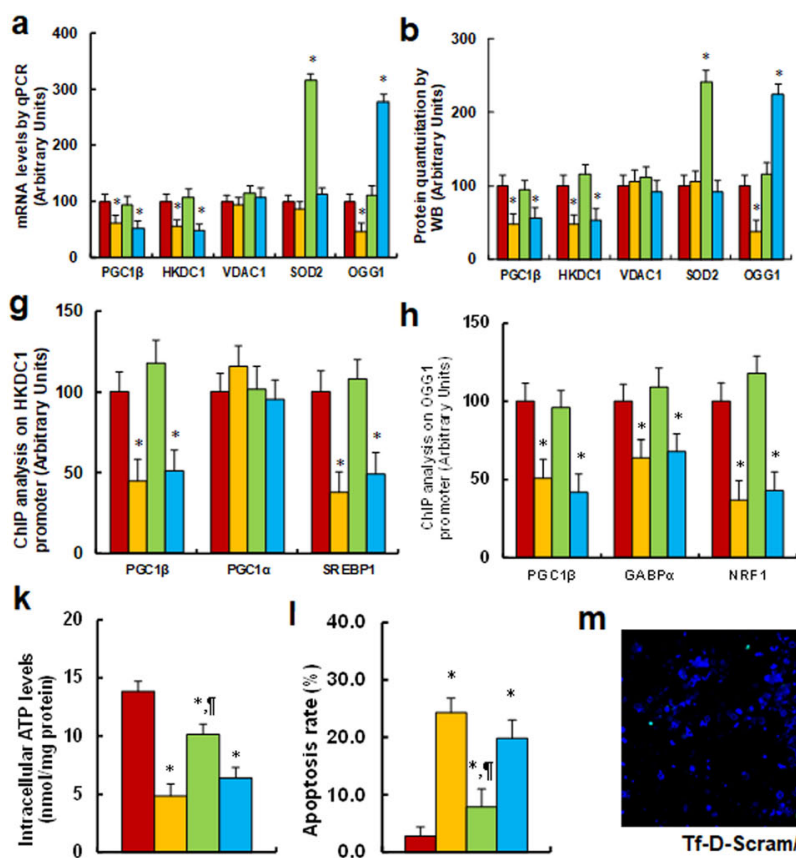

m

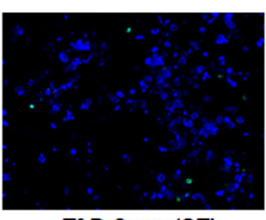

Tf-D-Scram/CTL

Fig. 4 Tf-D-HKC8 peptide-mediated HKDC1 interruption induces PGC1ß/OGG1 suppression and mitochondrial dysfunction. SNK6 cells were infected by SOD2, OGG1 or control (CTL) lentivirus and then treated with either scramble (Scram) or D-Tf-HKC8 peptide $(0.5 \mu \mathrm{M})$ for two days before being harvested for biomedical analysis. a) mRNA levels by $q P C R, n=4$. (b) Protein quantitation for Western Blotting. (c) Representative pictures for (b), $n=5$. (d) Representative pictures for IP/ WB. (e) Protein quantitation for (d), $n=5$. (f) Luciferase reporter
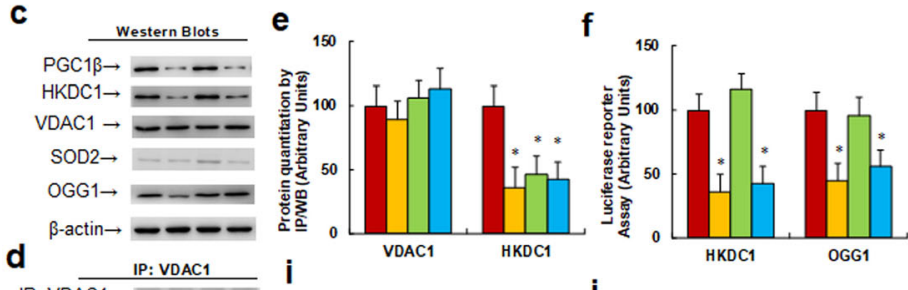

IB: VDAC1 $\rightarrow-$ IP: VDAC1

IB: HKDC1 $\rightarrow---$
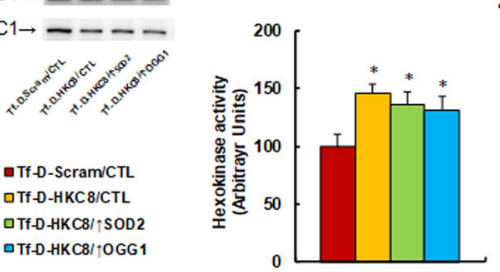

j
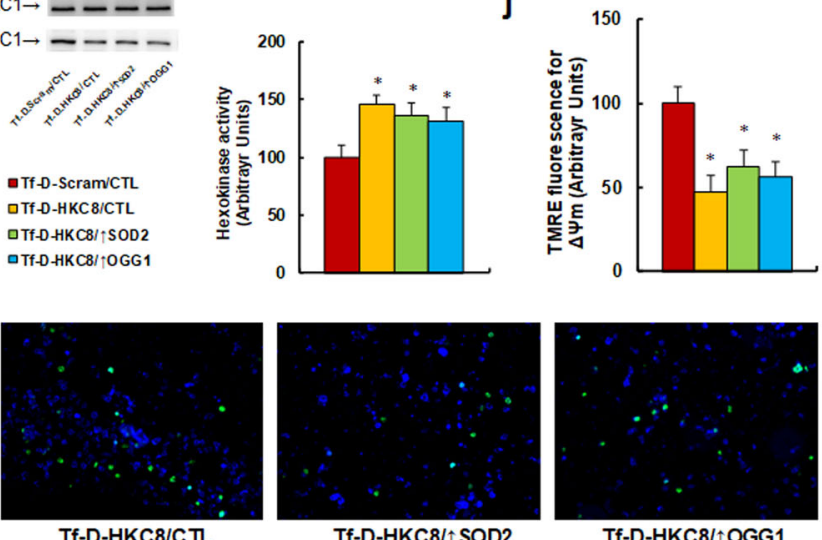

Tf-D-HKC8/CTL

Tf-D-HKC8/^SOD2

Tf-D-HKC8/^OGG1

activities for PGC1 $\beta$ and OGG1, $\mathrm{n}=5$. (g) ChIP analysis on HKDC1 promoter, $n=4$. (h) ChIP analysis on OGG1 promoter, $n=4$. (i). HKDC1 activity assay, $n=5$. (j) Mitochondrial membrane potential $(\Delta \Psi \mathrm{m}), \mathrm{n}=5$. (k) Intracellular ATP level, $n=5$. (l) Apoptosis rate by TUNEL assay, $\mathrm{n}=5$. (m) Representative pictures for (l). $*, P<0.05$, vs. Tf-D-Scram/ CTL group; II, $P<0.05$, vs. Tf-D-HKC $8 /$ CTL group. Data were expressed as mean \pm SEM 
OGG1, indicating successful peptide treatment and subsequent cellular internalization (Fig. 4i). Finally, we evaluated the effect of the Tf-D-HKC8 peptide on mitochondrial function and observed that treatment with Tf-D-HKC8/CTL decreased the mitochondrial membrane potential (MMP, $\triangle \Psi \mathrm{m}$ ) to $47 \%$, whereas no such effect was observed by the overexpression of either SOD2 or OGG1 (Fig. 4j). Furthermore, we found that the intercellular ATP levels were decreased to $35 \%$. Conversely, overexpression of SOD2 completely reversed this effect, whereas overexpression of OGG1 had no effect (Fig. 4k). In addition, treatment with Tf-D-HKC8/CTL increased the apoptosis rate to $868 \%$, with overexpression of SOD2 partly reversing this effect, whereas overexpression of OGG1 had no effect (Fig. 41, m). Our results indicated that the Tf-D-HKC8 peptide-mediated interruption of HKDC1 induced the suppression of PGC1 $\beta / O G G 1$ and mitochondrial dysfunction. This effect was completely and partly reversed with the overexpression of SOD2 and OGG1, respectively.

\section{Tf-D-HKC8 Peptide-mediated Interruption of HKDC1 Induced Oxidative Stress and Suppression of EBV}

We first evaluated the effect of SOD2 on Tf-D-HKC8 peptide-induced oxidative stress. Our results showed that treatment with Tf-D-HKC8/CTL increased the generation of ROS to $234 \%$ (Fig. 5a) and the formation of 3 nitrotyrosine to $207 \%$ (Fig. $5 \mathrm{~b}$ ), compared with the TfD-Scram/CTL group. Interestingly, we noted that overexpression of SOD2 completely reversed this effect, whereas overexpression of OGG1 had no effect. We then evaluated the effect of SOD2 on Tf-D-HKC8 peptide-induced DNA damage and found that treatment with Tf-D-HKC8/ CTL increased the formation of $\gamma \mathrm{H} 2 \mathrm{AX}$ to $195 \%$, with overexpression of SOD2 but not OGG1 completely reversing this effect (Fig. 5c and d, S6a). In addition, treatment with Tf-D-HKC8/CTL was demonstrated to increase the formation of $8-\mathrm{OHdG}$ to $250 \%$ (Fig. $5 \mathrm{e}$ and $\mathrm{f}$ ) and the tail length in the comet assay to $197 \%$ (Fig. $5 \mathrm{~g}$, h), with overexpression of both SOD2 and OGG1 completely reversing this effect. We also evaluated the effect of the TfD-HKC8 peptide on the replication of EBV. We observed that treatment with Tf-D-HKC8/CTL decreased the amount of EBV genomic DNA copies to 20\% (Fig. 5i) and decreased the EBV-encoded RNA to $29 \%$, as indicated by an EBER assay (Fig. 5j, k), compared with the TfD-Scram/CTL group. Overexpression of SOD2 and OGG1 reversed this effect completely and partly, respectively. Finally, we evaluated the effect of the Tf-D-HKC8 peptide on EBV-related gene expression. Our results showed that treatment with Tf-D-HKC8/CTL significantly
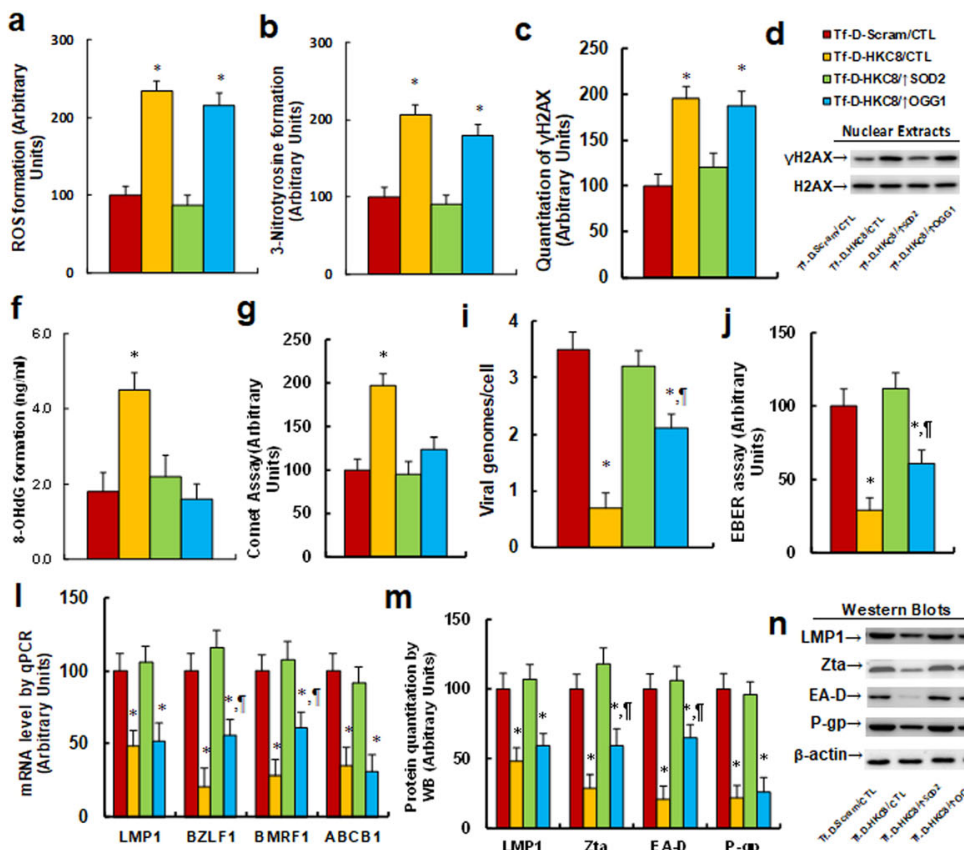

m
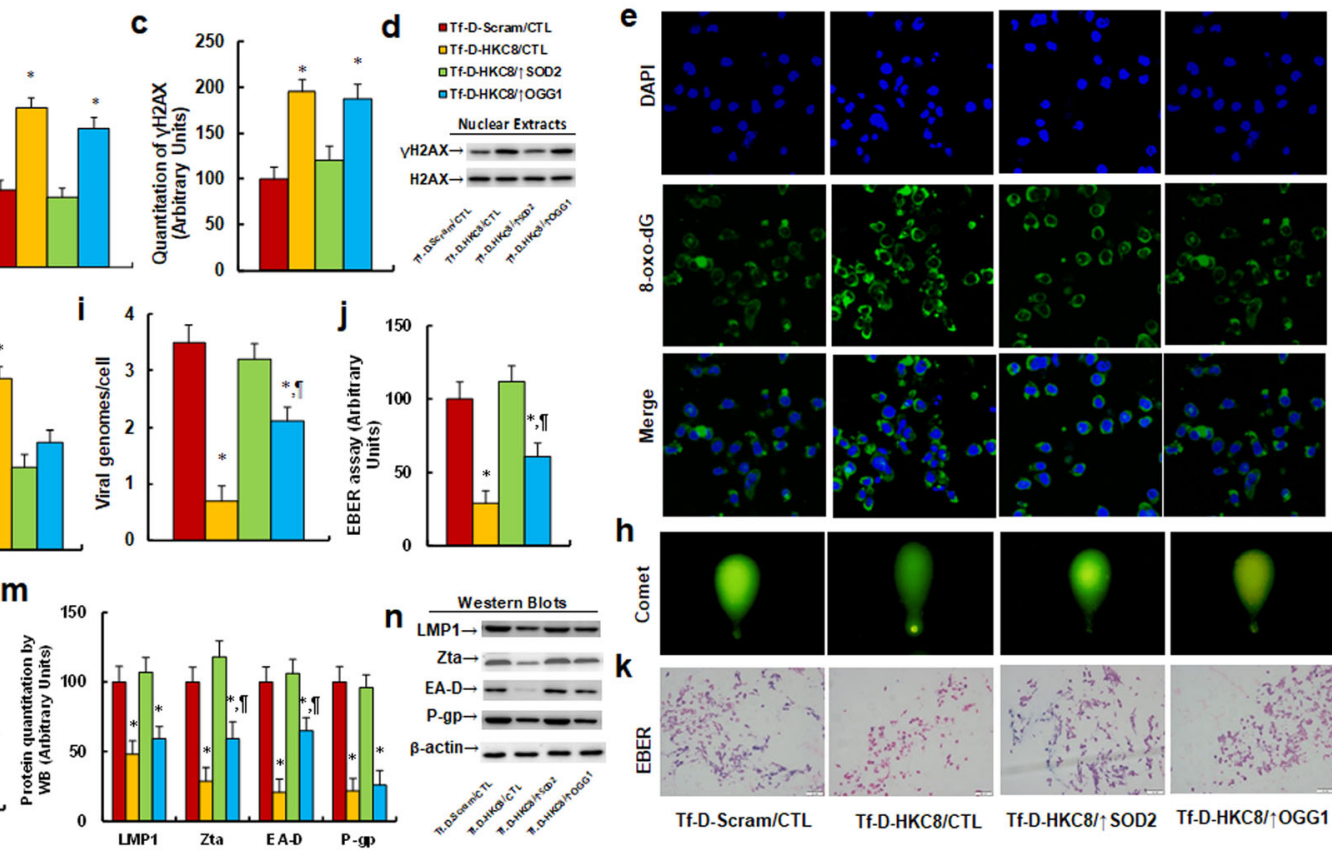

h
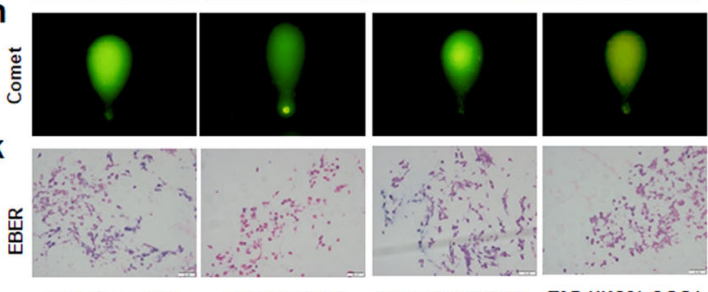

Tf-D-Scram/CTL Tf-D-HKC8/CTL Tf-D-HKC8/^SOD2 Tf-D-HKC8/^OGG1

Fig. 5 Tf-D-HKC8 peptide-mediated HKDC1 interruption induces oxidative stress and EBV suppression. SNK6 cells were infected by SOD2, OGG1 or control (CTL) lentivirus and then treated with either scramble (Scram) or D-Tf-HKC8 peptide $(0.5 \mu \mathrm{M})$ for two days before being harvested for biomedical analysis. a ROS formation, $n=5$. b 3nitrotyrosine (3-NT) formation, $\mathrm{n}=5$. $\mathbf{c}$ Quantitation of $\gamma \mathrm{H} 2 \mathrm{AX}$ formation, $\mathrm{n}=5$. $\mathbf{d}$ Representative pictures for $(\mathbf{c})$. e Representative pictures for 8-oxo-dG staining. f 8-OHdG formation, $\mathrm{n}=5$. g Quantitation of comet assay, $\mathrm{n}=4$. $\mathbf{h}$ Representative pictures for (g). i EBV viral genomes/cell by $q P C R, n=4$. $\mathbf{j}$ Quantitation for EBER assay, $n=4$. $\mathbf{k}$ Representative pictures for (j). I mRNA level by qPCR for BZLF1, BMRF1 and ABCB1, $\mathrm{n}=4$. $\mathrm{m}$ Protein quantitation for Zta, EA-D and P-gp, $\mathrm{n}=5$. n Representative pictures for $(\mathbf{m})$. $*, P<0.05$, vs. Tf-D-Scram/CTL group; II, $P<0.05$, vs. Tf-D-HKC $8 / \mathrm{CTL}$ group. Data were expressed as mean \pm SEM 
decreased the mRNA levels of LMP1, BZLF1, BMRF1, and $\mathrm{ABCB} 1$ compared with those in the Tf-D-Scram/CTL group. Conversely, overexpression of SOD2 was shown to completely reverse this effect, whereas overexpression of OGG1 partly restored the expression of BZLF1 and BMRF1, without affecting the expression of LMP1 or ABCB1 (Fig. 51). We then measured the protein levels of these genes and obtained an expression pattern similar to that of the mRNA levels (Fig. $5 \mathrm{~m}$ and n, S6b). Our results indicated that Tf-D-HKC8 peptide-mediated interruption of $\mathrm{HKDC} 1$ induced oxidative stress and suppression of EBV, which was completely and partly reversed by overexpression of SOD2 and OGG1, respectively.

\section{Tf-D-HKC8 Peptide-mediated Interruption of HKDC1 Suppressed Tumor Growth}

We evaluated the effect of SOD2 on Tf-D-HKC8 peptide-mediated tumor suppression. We found that treatment with Tf-D-HKC8/CTL decreased deoxyglucose uptake to $51 \%$ (Fig. 6a) and thymidine incorporation to $36 \%$ (Fig. 6b) compared with the Tf-D-Scram/CTL group. Whereas, overexpression of either SOD2 or OGG1 was shown to partly reverse this effect. Likewise, treatment with Tf-D-HKC8/CTL decreased colony formation to $31 \%$ (Fig. $6 \mathrm{c}$ and d), cell migration to $21 \%$ (Fig. $6 \mathrm{e}$ and $\mathrm{f}$ ), and the $\mathrm{Ki}-67$ positive cell rate to $29 \%$ (Fig. 6i and j) with overexpression of SOD2 and OGG1 completely and partly, reversing this effect, respectively. In addition, we noted that treatment with TfD-HKC8/CTL decreased cell invasion to $25 \%$; again, overexpression of SOD2 was demonstrated to completely reverse this effect, whereas overexpression of OGG1 had no effect on this process (Fig. 6g, h). Our results suggested that Tf-D-HKC8 peptide-mediated interruption of HKDC1 suppressed tumor growth with overexpression of SOD2 and OGG1 completely and partly reversing this effect, respectively.
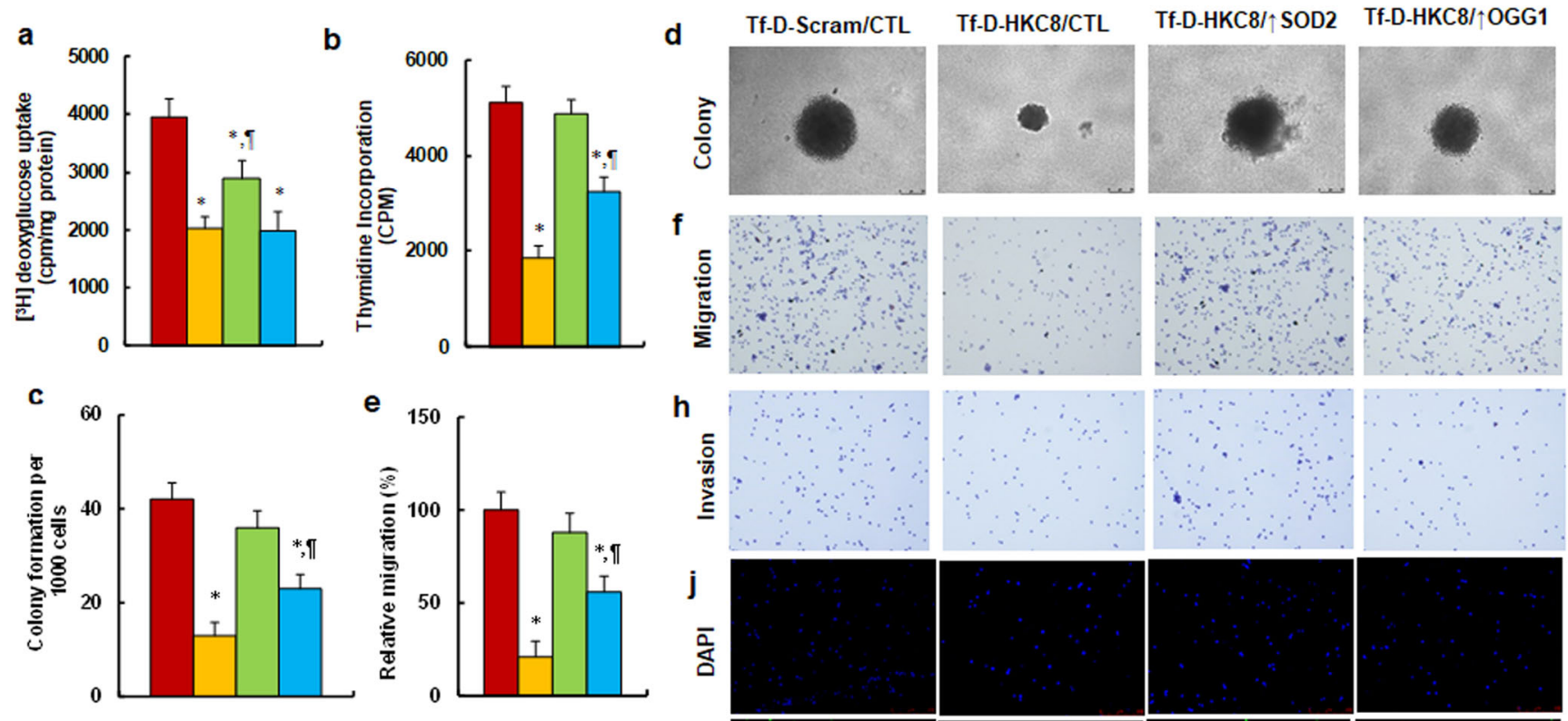

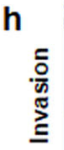
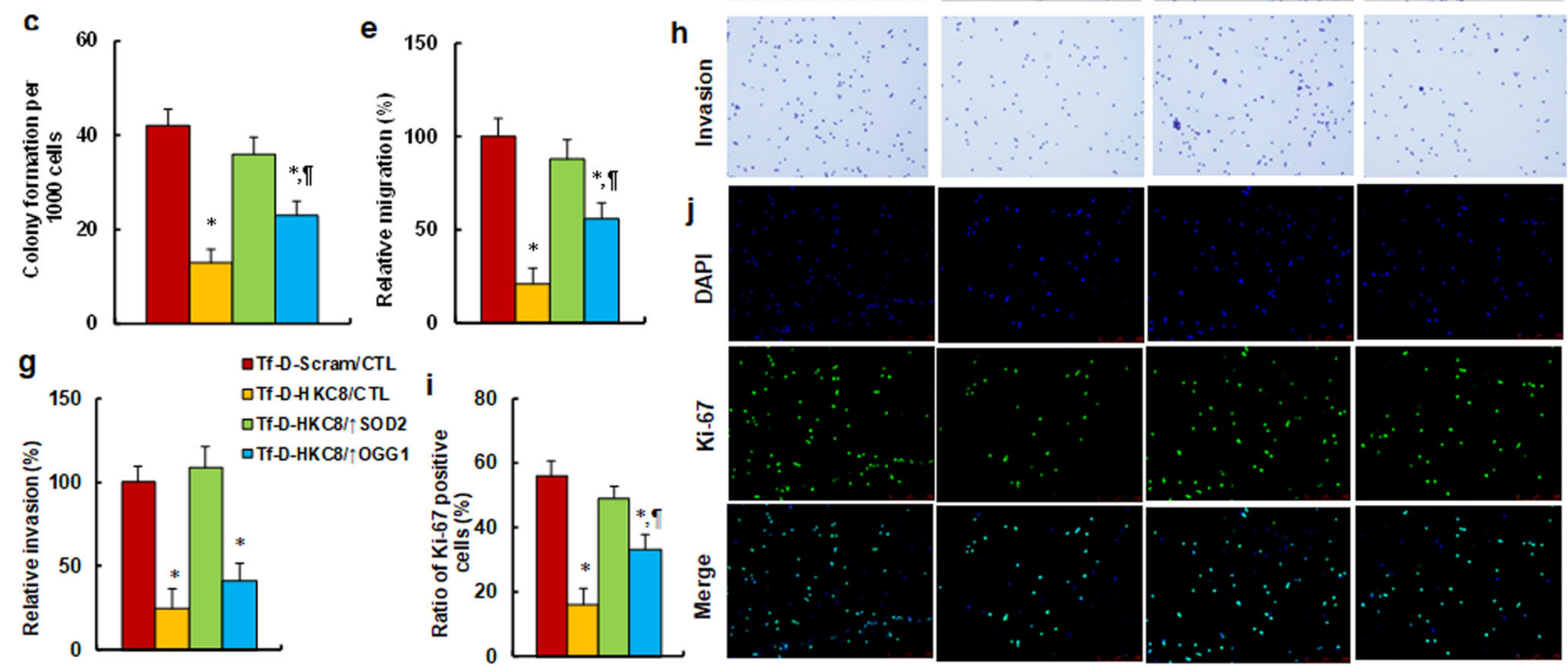

Fig. 6 Tf-D-HKC8 peptide-mediated HKDC1 interruption suppresses tumor growth. SNK6 cells were infected by SOD2, OGG1 or control (CTL) lentivirus and were then treated with either scramble (Scram) or D-Tf-HKC8 peptide $(0.5 \mu \mathrm{M})$ for 2 days before being harvested for biomedical analysis. a $\left[{ }^{3} \mathrm{H}\right]$ deoxyglucose uptake, $n=4$. b Cell proliferation analysis by thymidine incorporation, $\mathrm{n}=5$. c Colony formation assay

in soft agar, $n=4$. $\mathbf{d}$ Representative pictures for colony formation. e Cell migration assay, $\mathrm{n}=4$. $\mathbf{f}$ Representative pictures for $(\mathbf{e})$. $\mathbf{g}$ Cell invasion assay, $n=4$. h Representative pictures for (g). i Quantitation of Ki-67 positive cells, $\mathrm{n}=3$. j Representative picture for Ki-67 staining. *, $P<0.05$, vs. CTL group; II, $P<0.05$, vs. Tf-D-HKC 15 group. Data were expressed as mean \pm SEM 


\section{HKDC1 Knockdown Suppressed the PGC1ß/OGG1 Signaling Pathway in Addition to EBV Replication and Tumor Growth}

We evaluated the effect of LMP1 on tumor growth through the activation of PGC1 $\beta$ and the HKDC1 interruptionmediated tumor suppression through the generation of ROS in a mouse model. We injected nude mice with treated SNK6 cells through the tail vein. We then isolated the generated xenograft tumor tissues from the lungs for analysis, and calculated the survival rate. We first measured the protein expression in the PGC1 $\beta /$ OGG1 signaling pathway in tumor tissues and found that treatment with shHKDC1 (shHKDC1/EMP) significantly decreased the levels of the PGC1 $\beta$, HDKC1, and OGG1 proteins. Conversely, infection with a SOD2expressing lentivirus (shHKDC1/ $\uparrow \mathrm{SOD} 2$ ) completely reversed this effect, whereas an OGG1-expressing lentivirus (shHKDC1/ $\uparrow$ OGG1) had little effect, compared with the control group (CTL/EMP). Additionally, we observed that the levels of the VDAC1 protein were not altered under any of the treatments, whereas lentiviral infection with SOD2 and
OGG1 significantly increased the levels of each protein, indicating successful lentiviral manipulation of gene expression (Fig. 7a and b, S7a). We then evaluated the effect of the interruption of HKDC1 on EBV-related protein expression. Our results showed that treatment with $\mathrm{shHKDC} 1$ significantly decreased the levels of the LMP1, Zta (coded by BZLF1), EA-D (coded by BMRF1), and P-gp (coded by ABCB1) proteins compared with those in the CTL/EMP group. In this case too, we observed that overexpression of SOD2 completely reversed this effect, whereas overexpression of OGG1 partly restored the expression of LMP1 and Zta, but had no effect on EA-D and P-gp (Fig. 7c and d, S7b). We then evaluated the release of superoxide anion $\left(\mathrm{O}_{2}^{-}\right.$.) from xenograft tumor tissues and found that treatment with shHKDC1/EMP increased $\mathrm{O}_{2}^{-}$. release to $331 \%$ compared with the CTL/EMP group, with overexpression of SOD2 but not OGG1 completely reversing this effect (Fig. 7e). A similar effect was demonstrated on the amount of EBV genomic DNA copies, which were decreased to $16 \%$ (Fig. 7f). In addition, we noted that treatment with shHKDC1/EMP increased the formation of 8-oxo$\mathrm{dG}$ to $245 \%$, with overexpression of SOD2 partly, whereas
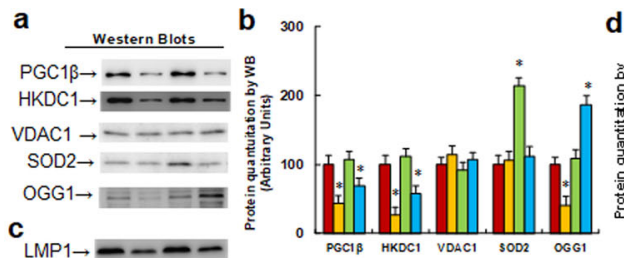

d
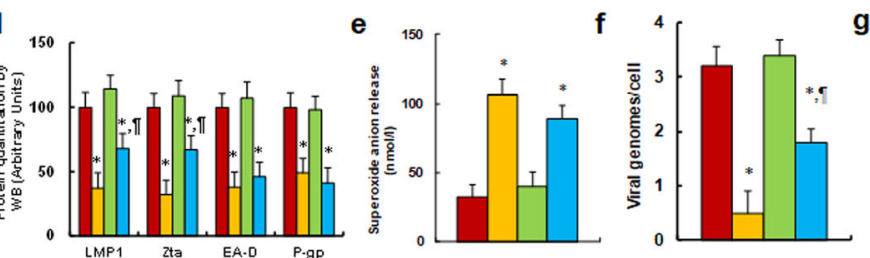

g
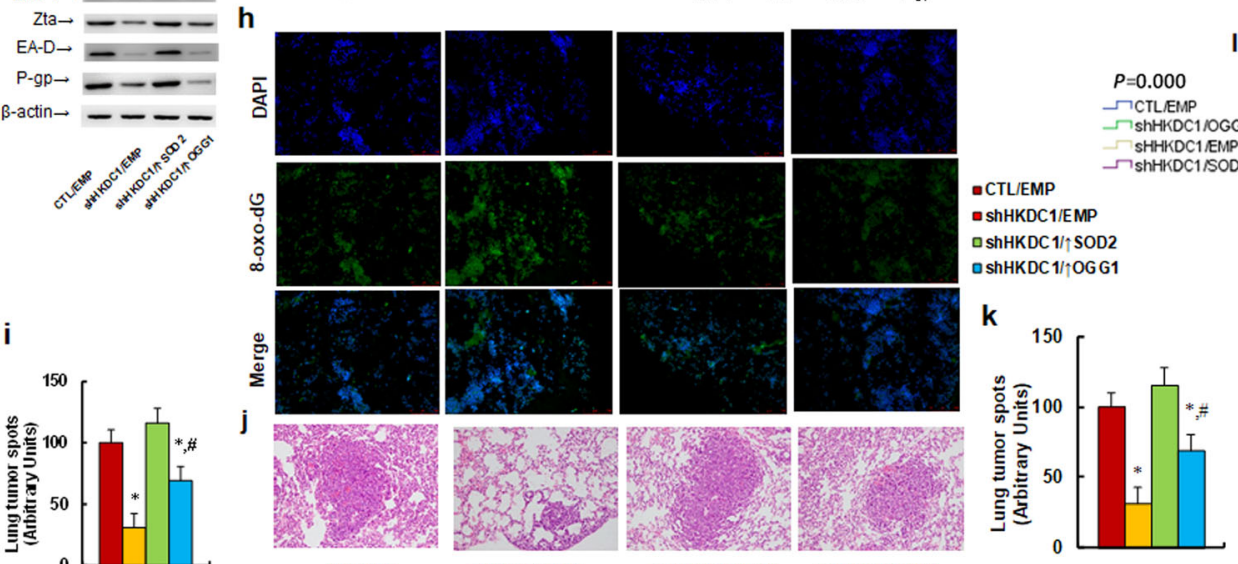

shHKDC1/^SOD2

shHKDC1/OGG1

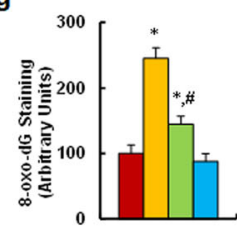

for (g). i-k Mice were killed upon $20 \%$ weight loss, and the lungs were harvested for terminal analysis. Metastatic tumor nodules from the lungs were counted, and then the formalin-fixed, paraffin-embedded tumor tissue of the lung was sectioned to $4 \mathrm{~mm}$ thickness before histopathological analyses were performed with $H \& E$ staining. Images were taken using a Carl Zeiss MIRAX MIDI slide scanner, and the lung tumor spots were analyzed using a 3DHISTECH Pannoramic Viewer. i Tumor colony formation in lung, $n=9$. $\mathbf{j}$ Representative picture by H\&E staining. k Quantitated lung tumor spots, $\mathrm{n}=5$. 1 Kaplan-Meier analysis comparing survival of mice between each treatment group, $P$ value represents logrank Mantel-Cox test result, $\mathrm{n}=9$. ${ }^{*}, P<0.05$, vs. CTL/EMP group; II, $P<0.05$, vs. shHKDC1/EMP group. Data were expressed as mean \pm SEM 
OGG1 completely reversing this effect (Fig. $7 \mathrm{~g}, \mathrm{~h}$ ). Subsequently, we investigated the effect of treatments on tumor nodule formation and showed that treatment with shHKDC1/EMP decreased tumor colony formation in the lung to $32 \%$ (Fig. 7i) and lung tumor spots to $31 \%$, as indicated by H\&E staining (Fig. 7j, k) These effects were shown to be completely and partly reversed by overexpression of SOD2 and OGG1, respectively. Finally, we evaluated the effects of treatments on mouse survival rates using KaplanMeier analysis. Our results showed that treatment with shHKDC1/EMP significantly increased mouse survival rates compared with that for the CTL/EMP group. Once again, overexpression of SOD2 and OGG1 completely and partly diminished this effect, respectively (Fig. 71). Our results suggested that knocking-down HKDC1 suppressed the PGC1 $\beta$ / OGG1 signaling pathway in addition to the replication of EBV and tumor growth in a mouse model. These effects were completely and partly reversed by the overexpression of SOD2 and OGG1, respectively.

\section{Schematic Model of the EBV LMP1-induced PGC1 $\beta$ Signaling Pathway and Tumor Growth}

We created a schematic model illustrating the LMP1mediated tumor development through the activation of PGC1 $\beta$ and tumor suppression through the interruption of HKDC1 (Fig. 8). According to this model, the EBVencoded LMP1 protein activates NF-KB, causing subunits p65 and p50 to bind to the NF- $\mathrm{KB}$ responsive element located on the PGC1 $\beta$ promoter, thus increasing the expression of PGC1 $\beta$. Subsequently, the upregulated PGC1 $\beta$ further increases the expression of HKDC1 through the coactivation of SREBP1 and the expression of OGG1 through the coactivation of NRF1 and GABP $\alpha$. The upregulated HKDC1 leads to increased mitochondrial function through its binding with VDAC1, favoring glycolysis and tumor growth. Concomitantly, the upregulation of OGG1 minimizes the generation of 8-oxo-dG, and increases DNA repair to prevent ROS-induced DNA damage in the EBV genome (shown in red circles). Whereas, interruption of HKDC1 through either the Tf-D-HKC8 peptide or shRNA inhibits the binding of HKDC1 and VDAC1, triggering mitochondrial dysfunction and generation of ROS. Increased generation of ROS results in direct EBV DNA damage with suppression of LMP1 and subsequent suppression of the $\mathrm{PGC} 1 \beta$ signaling pathway and tumor growth (shown in blue circle). In contrast, overexpression of either SOD2 or OGG1 suppresses oxidative stressinduced EBV DNA damage, thus preventing the HKDC1 interruption-induced suppression of EBV and tumor growth.

\section{Discussion}

In this study, we found that the expression of LMP1 upregulated the expression of PGC1 $\beta$ through activation of NF-KB, with activated PGC1 $\beta$ upregulating the expression of OGG1

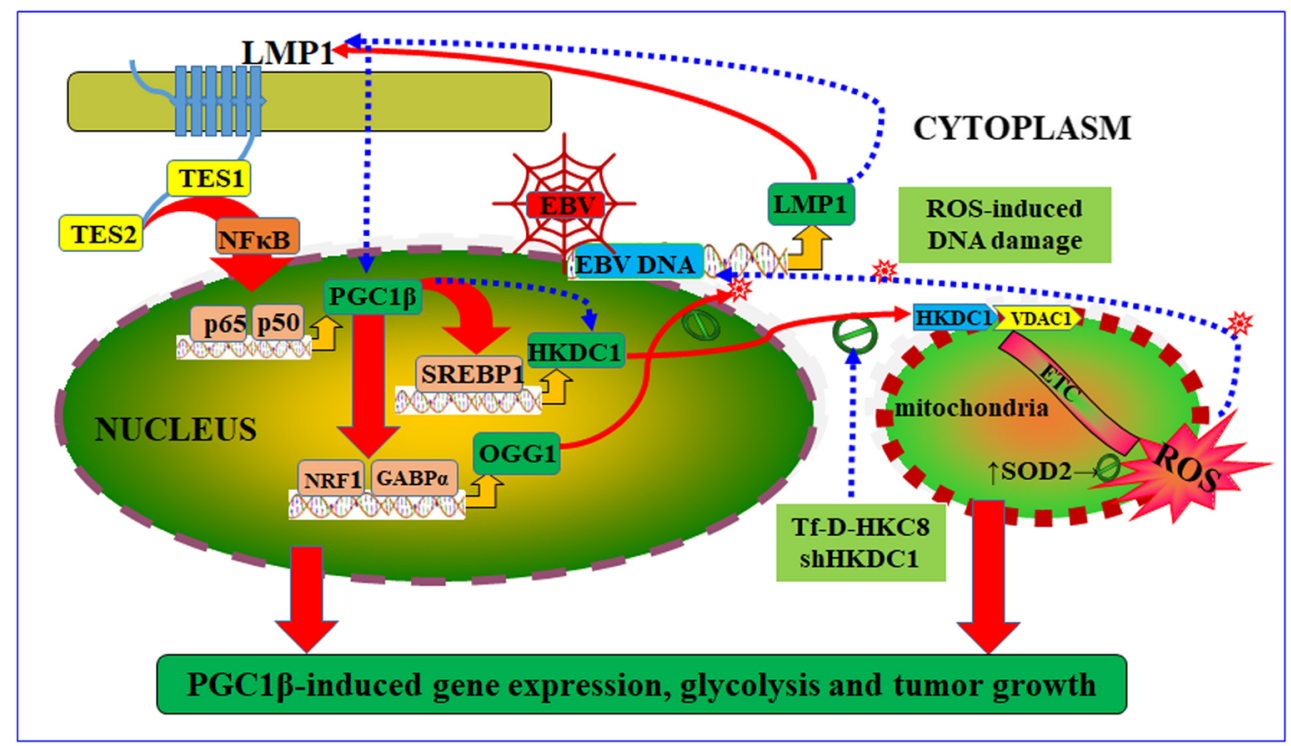

Fig. 8 Schematic model for EBV LMP1-induced PGC1 $\beta$ signaling pathway and tumor growth. Abbreviations: EBV: Epstein-Barr virus; ETC: electron transport chain; GABP $\alpha$ : GA-binding protein $\alpha$; HKDC1: Hexokinase domain component 1; LMP1: latent membrane protein 1; NRF1: (nuclear respiratory factor 1 ); NF- $\mathrm{kB}$ : nuclear factorKB; OGG1: 8-oxoguanine DNA glycosylase 1; PGC1 $\beta$ : PGC1 $\beta$ : peroxisome proliferator-activated receptor- $\gamma(\operatorname{PPAR} \gamma)$ coactivator- $1 \beta$; ROS; reactive oxygen species; SOD2: superoxide dismutase 2; TES: transformation effector site; Tf-D-HKC8: last 8 amino acid of $\mathrm{HKDC} 1$ at the C-terminal (HKC8) with D-configuration using transferrin (Tf) receptor internalization sequence; VDAC1: vascular endothelial growth factor 1 
through coactivation of NRF1 and GABP $\alpha$. We further found that a HKDC1 interruption-mediated generation of ROS triggered EBV DNA damage, resulting in LMP1-mediated suppression of the PGC1 $\beta / \mathrm{HKDC} 1 / \mathrm{OGG} 1$ signaling pathway, subsequently suppressing EBV-associated tumorigenesis.

In order to evaluate the gene expression in NKTCL cells, we selected T-lymphocytes from peripheral blood as the control. While T-lymphocytes were relatively few, they were sufficient for mRNA analysis, but not enough for western blotting. In this case, we chose peripheral blood mononuclear cells (PBMCs) as the alternative control for gene analysis. Our results showed that the mRNA expression was not significantly different between T-lymphocytes and PBMCs, and thus PBMCs were finally chosen as a control throughout this study. Our results showed that the Tf-D-HKC8 peptide blocked the binding of HKDC1 with VDAC1, triggering the release of mitochondrial superoxide, resulting in subsequent oxidative stress. In order to specifically block the Tf-D-HKC8 peptide-mediated mitochondrial oxidative stress, we used the SOD2-expressing lentivirus as a ROS scavenger throughout this study. The SOD2 protein is known to convert mitochondrial superoxide into the less toxic hydrogen peroxide, and unlike other ROS scavengers, such as SOD1, which are mostly located in the cytosol, SOD2 is also found in the mitochondria. In addition, catalase, which is known to detoxify hydrogen peroxide, has little effect on mitochondrial superoxide, while N-acetyl cysteine (NAC), which is a general antioxidant, can only transiently scavenge a certain amount of ROS and cannot kinetically scavenge ROS when shortly oxidized. Based on these observations, the SOD2 mitochondrial ROS scavenger was the best choice for this study, as previously mentioned [37, 38].

Our results showed that the expression of LMP1 and PGC1 $\beta$ was significantly increased in different NKTCL cells compared with healthy PBMCs. Additionally, we found that infection with LMP1-expressing lentivirus significantly increased the PGC1 $\beta$ luciferase reporter activity, with the LMP1-responsive element on the PGC1 $\beta$ promoter being identified as the one for the NF- $\mathrm{KB}$ transcription factor. Accordingly, the expression of LMP1 was significantly increased in healthy PBMCs, whereas knocking-down LMP1 in NKTCL cells significantly decreased the ability of NF-KB p65 to bind to the PGC1 $\beta$ promoter. Furthermore, we noted that a NF- $\mathrm{kB}$ knockdown of either subunit $\mathrm{p} 65$ or p50 significantly decreased the expression of PGC1 $\beta$. These results provided very powerful evidence for the upregulation of PGC1 $\beta$ by EBV LMP1 through the activation of NF- $K B$. Many studies have reported the LMP1-mediated oncogenicity through the promotion of glycolysis and activation of NF- $\mathrm{KB}$ $[12-17,39]$, the detailed mechanism remains largely unknown. This study provided a bridge linking the mechanisms of the activation of LMP1/NF- $\mathrm{KB}$ and tumor development through the activation of $\mathrm{PGC} 1 \beta$. In addition, our preliminary data showed that many EBV-associated tumors with a highly upregulated expression of PGC1 $\beta$, did not have active LMP1 protein. We assumed that transient exposure to LMP1 might trigger epigenetic changes on the $\mathrm{PGC} 1 \beta$ promoter, resulting in the long-lasting upregulation of PGC1 $\beta$ during the subsequent latent stage of LMP1 [40]. Therefore, targeting PGC1 $\beta$ might become more attractive for tumor development than targeting LMP1 in the latent stage and we are currently investigating this [41].

Our study showed that the expression of PGC1 $\beta$ and OGG1 was significantly increased in different NKTCL cells compared with healthy PBMCs. Additionally, we found that the expression of PGC1 $\beta$ significantly increased the OGG1 luciferase reporter activity, and identified the NRF1 and GABP $\alpha$ binding sites as the PGC1 $1 \beta$-responsive elements on the OGG1 promoter. The expression of PGC1 $\beta$ was significantly increased in healthy PBMCs, whereas knocking-down PGC1 $\beta$ significantly decreased the binding ability of both $\mathrm{NRF} 1$ and $\mathrm{GABP} \alpha$ to the $\mathrm{PGC} 1 \beta$ promoter. As knockdown of either NRF1 or GABP $\alpha$ significantly decreased the expression of OGG1, we concluded that its expression was regulated by PGC1 $\beta$ through the coactivation of NRF1 and GABP $\alpha$. The expression of OGG1 was shown to minimize the ROSmediated DNA damage, which is very important for the survival of EBV DNA, as it is relatively naked compared with histone-wrapped somatic DNA, making it more susceptible to attacks by ROS, especially during the replication process. In case that the ROS-mediated base incision cannot be repaired in time, the EBV DNA will eventually be destructed or lose its potency $[35,42-44]$. However, the PGC1 $\beta$ expressionmediated upregulation of OGG1 was demonstrated to protect the EBV genome from ROS-mediated DNA damage, favoring EBV DNA survival from the mitochondrial dysfunctionmediated generation of ROS. Additionally, we recently found that $\mathrm{PGC} 1 \beta$ promoted glycolysis metabolism through the activation of LDHA [25]. Furthermore, it was shown to upregulate the expression of $\mathrm{HKDC} 1$ through the activation of SREBP1, resulting in increased mitochondrial function and glucose metabolism, thus favoring tumor growth [26]. In addition, we also found that $\mathrm{PGC} 1 \beta$ promoted mitochondrial biogenesis and glycolysis metabolism [20-23]. Taken together, we concluded that the LMP1-mediated expression of PGC1 $\beta$ promoted EBV-mediated tumorigenesis, in particular by favoring EBV DNA survival through the activation of OGG1.

We further showed that interruption of HKDC1 by either shHKDC1 or Tf-D-HKC8 peptide [36] led to the decreased interaction of HKDC11 with VDAC1 [45], triggering mitochondrial dysfunction, generation of ROS, and ROS-mediated DNA damage. Surprisingly, the interruption of HKDC1 was also shown to inhibit the expression of LMP1 and its downstream target genes, including PGC1 $\beta$, OGG1, and HKDC1 itself as well as EBV-related proteins, including Zta, EA-D, 
and P-gp [6, 7, 46, 47]. However, we observed that overexpression of SOD2 and OGG1 reversed this effect completely and partly, respectively, indicating that the HKDC1 interruption-mediated suppression of EBV and target genes was primarily due to the generation of ROS and ROSmediated EBV DNA damage. Taken together, we demonstrated that the HKDC1 interruption-mediated generation of ROS suppressed the EBV genome through the induction of DNA damage, resulting in the suppression of LMP1 and its target genes, including PGC1 $1 \beta$, OGG1, and HKDC1 itself. Consequently, the suppression of HKDC1 was shown to trigger further mitochondrial dysfunction and generation of ROS, providing a positive feedforward loop for the generation of ROS and subsequent suppression of the EBV genome.

Our results showed that interruption of HKDC1 by the TfD-HKC8 peptide [36] in NKTCL cells significantly suppressed the cellular deoxyglucose uptake, colony formation, cell migration and invasion, and cell proliferation in vitro. Furthermore, we observed that interruption of HKDC1 by shHKDC1 significantly suppressed in vivo tumor formation and increased mouse survival; overexpression of SOD2 completely, whereas OGG1 only partly, reversed this effect, suggesting that the HKDC1 interruption-mediated antitumor effect was due to the generation of ROS and subsequent ROSmediated DNA damage. Furthermore, our previous studies showed that the level of the expression of HKDC1 was significantly increased in NKTCL cells, whereas that was not the case in healthy PBMCs, which exhibited very low levels [26, 36]. These findings make HKDC1 a very attractive target for the development of anti-EBV and antitumor drugs, as interruption of $\mathrm{HKDC} 1$ was shown to not significantly affect normal cells. As our data indicated that the EBV genome seems to be a potential driving force for tumorigenesis in NKTCL cells through the LMP1-mediated PGC1 $\beta / \mathrm{HKDC1/OGG1} \mathrm{signal-}$ ing pathway $[26,32,33]$ and targeting $\mathrm{HKDC} 1$ is especially efficient in NKTCL cells, the development of novel molecules targeting $\mathrm{HKDC} 1$ is currently under investigation.

\section{Conclusions}

LMP1 is the potential driving force for EBV-mediated tumorigenesis through activation of the PGC1 $\beta / \mathrm{HKDC} 1 / \mathrm{OGG} 1$ signaling pathway. Furthermore, HKDC1 interruptionmediated mitochondrial dysfunction forms a positive feed forward loop for ROS outbreak, subsequently suppressing EBV replication and LMP1/PGC1 $\beta$ signaling pathway-mediated tumor growth. Taken altogether, this study provides a novel therapeutic strategy for targeting HKDC1 for NKTCL treatment.

Supplementary Information The online version contains supplementary material available at https://doi.org/10.1007/s12015-020-10112-8.
Author Contributions P.Y. wrote the paper; P.Y. and H.Z. designed the experiments, analyzed the data and interpreted the results; P. Z. performed the parts of the gene analysis and IHC staining experiments. X.H. and W.X. performed the parts of the experiments for plasmid construction and lentivirus. J.F., and Q.C. performed the remaining experiments. All authors read and approved the final manuscript.

Funding This study was financially supported by The National Natural Science Foundation of China, Project \#: 81772097 \& 31601172; Shenzhen SanMing Project \#: SZSM201612004; Shenzhen Science and Technology Planning Project \#: JCYJ20170816105345191; Shenzhen Science and Technology Innovation Committee International Cooperation Project \#: GJHZ20180420180752400; Guangdong Science and Technology Program \#: 2017ZC0398; Natural Science Foundation of Guangdong Province \#: 2016A030310069; Medical Science and Technology Research Foundation of Guangdong Province \#: A2019540; Shenzhen Science and Technology Innovation Committee Basic Science Research Grant \#: JCYJ20170411090739316 \& JCYJ20170306161807726 and Shenzhen High-level Hospital Construction Fund.

\section{Compliance with Ethical Standards}

Ethical Approval and Consent to Participate The animal protocol conformed to US NIH guidelines (Guide for the Care and Use of Laboratory Animals, No. 85-23, revised 1996), and was reviewed and approved by the Institutional Animal Care and Use Committee from Peking University Shenzhen Hospital. The human study was carried out in accordance with the recommendations of the Human Subject Guidelines of the Human Subjects Institutional Review Board. The protocol was approved by Peking University Shenzhen Hospital. All subjects gave written informed consent in accordance with the Declaration of Helsinki.

Conflict of Interest The authors declare that they have no competing interests.

Abbreviations EBV, Epstein-Barr virus; GABP $\alpha$, GA-binding protein $\alpha$ (NRF2); HKDC1, Hexokinase domain component 1; LMP1, latent membrane protein 1 ; MMP, mitochondrial membrane potential $(\Delta \Psi \mathrm{m})$; NRF1, nuclear respiratory factor 1 ; NF- $\mathrm{KB}$, nuclear factor-kB; NKTCL, Natural killer/T-cell lymphoma; OGG1, 8-oxoguanine DNA glycosylase $1 ; \mathrm{O}_{2}{ }^{-}$, superoxide anions; PGC1 $\beta$, peroxisome proliferator-activated receptor- $\gamma(\mathrm{PPAR} \gamma)$ coactivator-1 $\beta$; P-gp, P-glycoprotein; ROS, reactive oxygen species; SOD2, superoxide dismutase 2; VDAC1, vascular endothelial growth factor 1

Open Access This article is licensed under a Creative Commons Attribution 4.0 International License, which permits use, sharing, adaptation, distribution and reproduction in any medium or format, as long as you give appropriate credit to the original author(s) and the source, provide a link to the Creative Commons licence, and indicate if changes were made. The images or other third party material in this article are included in the article's Creative Commons licence, unless indicated otherwise in a credit line to the material. If material is not included in the article's Creative Commons licence and your intended use is not permitted by statutory regulation or exceeds the permitted use, you will need to obtain permission directly from the copyright holder. To view a copy of this licence, visit http://creativecommons.org/licenses/by/4.0/. 


\section{References}

1. Dufva, O., Kankainen, M., Kelkka, T., Sekiguchi, N., Awad, S. A., Eldfors, S., et al. (2018). Aggressive natural killer-cell leukemia mutational landscape and drug profiling highlight JAK-STAT signaling as therapeutic target. Nature Communications, 9(1), 1567.

2. Yang, Y., Zhu, Y., Cao, J. Z., Zhang, Y. J., Xu, L. M., Yuan, Z. Y., et al. (2015). Risk-adapted therapy for early-stage extranodal nasaltype NK/T-cell lymphoma: analysis from a multicenter study. Blood, 126(12), 1424-1432. quiz 517.

3. Jiang, L., Gu, Z. H., Yan, Z. X., Zhao, X., Xie, Y. Y., Zhang, Z. G., et al. (2015). Exome sequencing identifies somatic mutations of DDX3X in natural killer/T-cell lymphoma. Nature Genetics, 47(9), 1061-1066.

4. de Mel, S., Hue, S. S., Jeyasekharan, A. D., Chng, W. J., \& Ng, S. B. (2019). Molecular pathogenic pathways in extranodal NK/T cell lymphoma. Journal of Hematology \& Oncology, 12(1), 33.

5. Kwong, Y. L., Chan, T. S. Y., Tan, D., Kim, S. J., Poon, L. M., Mow, B., et al. (2017). PD1 blockade with pembrolizumab is highly effective in relapsed or refractory NK/T-cell lymphoma failing 1asparaginase. Blood, 129(17), 2437-2442.

6. Darby, R. A., Callaghan, R., \& McMahon, R. M. (2011). Pglycoprotein inhibition: the past, the present and the future. Current Drug Metabolism, 12(8), 722-731.

7. Yoshimori, M., Takada, H., Imadome, K., Kurata, M., Yamamoto, K., Koyama, T., et al. (2015). P-glycoprotein is expressed and causes resistance to chemotherapy in EBV-positive T-cell lymphoproliferative diseases. Cancer Medicine, 4(10), 1494-1504.

8. Tse, E., \& Kwong, Y. L. (2017). The diagnosis and management of NK/T-cell lymphomas. Journal of Hematology \& Oncology, 10(1), 85.

9. Yong, W. (2016). Clinical study of 1-asparaginase in the treatment of extranodal NK/T-cell lymphoma, nasal type. Hematological Oncology, 34(2), 61-68.

10. Nam, Y. S., Im, K. I., Kim, N., Song, Y., Lee, J. S., Jeon, Y. W., et al. (2019). Down-regulation of intracellular reactive oxygen species attenuates P-glycoprotein-associated chemoresistance in Epstein-Barr virus-positive NK/T-cell lymphoma. American Journal of Translational Research, 11(3), 1359-1373.

11. Zhang, H., Lu, J., Jiao, Y., Chen, Q., Li, M., Wang, Z., et al. (2018). Aspirin inhibits natural killer/T-cell lymphoma by modulation of VEGF expression and mitochondrial function. Frontiers in Oncology, 8, 679.

12. Chou, Y. C., Lin, S. J., Lu, J., Yeh, T. H., Chen, C. L., Weng, P. L., et al. (2011). Requirement for LMP1-induced RON receptor tyrosine kinase in Epstein-Barr virus-mediated B-cell proliferation. Blood., 118(5), 1340-1349.

13. Wang, L. W., Jiang, S., \& Gewurz, B. E. (2017). Epstein-barr virus LMP1-mediated oncogenicity. Journal of Virology, 91(21), e01718-16.

14. Higuchi, M., Izumi, K. M., \& Kieff, E. (2001). Epstein-Barr virus latent-infection membrane proteins are palmitoylated and raft-associated: protein 1 binds to the cytoskeleton through TNF receptor cytoplasmic factors. Proceedings of the National Academy of Sciences of the United States of America, 98(8), 4675-4680.

15. Dudziak, D., Kieser, A., Dirmeier, U., Nimmerjahn, F., Berchtold, S., Steinkasserer, A., et al. (2003). Latent membrane protein 1 of Epstein-Barr virus induces CD83 by the NF-kappaB signaling pathway. Journal of Virology, 77(15), 8290-8298.

16. Cahir McFarland, E. D., Izumi, K. M., \& Mosialos, G. (1999). Epstein-barr virus transformation: involvement of latent membrane protein 1-mediated activation of NF-kappaB. Oncogene., 18(49), 6959-6964.
17. Perkins, N. D. (2012). The diverse and complex roles of NFkappaB subunits in cancer. Nature Reviews. Cancer, 12(2), 121132.

18. Lin, J., Puigserver, P., Donovan, J., Tarr, P., \& Spiegelman, B. M (2002). Peroxisome proliferator-activated receptor gamma coactivator 1 beta (PGC-1 beta), a novel PGC-1-related transcription coactivator associated with host cell factor. The Journal of Biological Chemistry, 277(3), 1645-1648.

19. Lin, J., Handschin, C., \& Spiegelman, B. M. (2005). Metabolic control through the PGC-1 family of transcription coactivators. Cell Metabolism, 1(6), 361-370.

20. Chang, C. Y., Kazmin, D., Jasper, J. S., Kunder, R., Zuercher, W. J., \& McDonnell, D. P. (2011). The metabolic regulator ERRalpha, a downstream target of HER2/IGF-1R, as a therapeutic target in breast cancer. Cancer Cell., 20(4), 500-510.

21. Deblois, G., Chahrour, G., Perry, M. C., Sylvain-Drolet, G., Muller, W. J., \& Giguere, V. (2010). Transcriptional control of the ERBB2 amplicon by ERRalpha and PGC-1beta promotes mammary gland tumorigenesis. Cancer Research, 70(24), 10277-10287.

22. Bellafante, E., Morgano, A., Salvatore, L., Murzilli, S., Di Tullio, G., D’Orazio, A., et al. (2014). PGC-1beta promotes enterocyte lifespan and tumorigenesis in the intestine. Proceedings of the National Academy of Sciences of the United States of America, 111(42), E4523-E4531.

23. Deblois, G., St-Pierre, J., \& Giguere, V. (2013). The PGC-1/ERR signaling axis in cancer. Oncogene., 32(30), 3483-3490.

24. Piccinin, E., Villani, G., \& Moschetta, A. (2019). Metabolic aspects in NAFLD, NASH and hepatocellular carcinoma: the role of PGC1 coactivators. Nature Reviews. Gastroenterology \& Hepatology, 16(3), 160-174.

25. Zhang, H., Li, L., Chen, Q., Li, M., Feng, J., Sun, Y., et al. (2018). PGC1beta regulates multiple myeloma tumor growth through LDHA-mediated glycolytic metabolism. Molecular Oncology, 12(9), 1579-1595.

26. Chen, X., Lv, Y., Sun, Y., Zhang, H., Xie, W., Zhong, L., et al. (2019). PGC1beta regulates breast tumor growth and metastasis by SREBP1-mediated HKDC1 expression. Frontiers in Oncology, 9, 290.

27. Khan, M. W., Ding, X., Cotler, S. J., Clarke, M., \& Layden, B. T. (2018). Studies on the tissue localization of HKDC1, a putative novel fifth hexokinase, in humans. The Journal of Histochemistry and Cytochemistry, 66(5), 385-392.

28. Majewski, N., Nogueira, V., Bhaskar, P., Coy, P. E., Skeen, J. E., Gottlob, K., et al. (2004). Hexokinase-mitochondria interaction mediated by Akt is required to inhibit apoptosis in the presence or absence of Bax and Bak. Molecular Cell, 16(5), 819-830.

29. Patra, K. C., Wang, Q., Bhaskar, P. T., Miller, L., Wang, Z., Wheaton, W., et al. (2013). Hexokinase 2 is required for tumor initiation and maintenance and its systemic deletion is therapeutic in mouse models of cancer. Cancer Cell., 24(2), 213-228.

30. Ludvik, A. E., Pusec, C. M., Priyadarshini, M., Angueira, A. R., Guo, C., Lo, A., et al. (2016). HKDC1 is a novel hexokinase involved in whole-body glucose use. Endocrinology., 157(9), 34523461.

31. Guo, C., Ludvik, A. E., Arlotto, M. E., Hayes, M. G., Armstrong, L. L., Scholtens, D. M., et al. (2015). Coordinated regulatory variation associated with gestational hyperglycaemia regulates expression of the novel hexokinase HKDC1. Nature Communications, 6, 6069.

32. Zhang, Z., Huang, S., Wang, H., Wu, J., Chen, D., Peng, B., et al. (2016). High expression of hexokinase domain containing 1 is associated with poor prognosis and aggressive phenotype in hepatocarcinoma. Biochemical and Biophysical Research Communications, 474(4), 673-679.

33. Fuhr, L., El-Athman, R., Scrima, R., Cela, O., Carbone, A., Knoop, H., et al. (2018). The circadian clock regulates metabolic phenotype 
rewiring via $\mathrm{HKDC} 1$ and modulates tumor progression and drug response in colorectal cancer. EBioMedicine., 33, 105-121.

34. Chen, Q., Feng, J., Wu, J., Yu, Z., Zhang, W., Chen, Y., et al. (2020). HKDC1 C-terminal based peptides inhibit extranodal natural killer/T-cell lymphoma by modulation of mitochondrial function and EBV suppression. Leukemia., 34(10), 2736-2748.

35. David, S. S., O'Shea, V. L., \& Kundu, S. (2007). Base-excision repair of oxidative DNA damage. Nature., 447(7147), 941-950.

36. Chen, Q., Feng, J., Wu, J., Yu, Z., Zhang, W., Chen, Y., et al. (2020). HKDC1 C-terminal based peptides inhibit extranodal natural killer/T-cell lymphoma by modulation of mitochondrial function and EBV suppression. Leukemia, 34, 2736-2748.

37. Wang, X., Lu, J., Xie, W., Lu, X., Liang, Y., Li, M., et al. (2019). Maternal diabetes induces autism-like behavior by hyperglycemiamediated persistent oxidative stress and suppression of superoxide dismutase 2. Proceedings of the National Academy of Sciences of the United States of America, 116(47), 23743-23752.

38. Brownlee, M. (2001). Biochemistry and molecular cell biology of diabetic complications. Nature., 414(6865), 813-820.

39. Meckes, D. G., Jr., Menaker, N. F., \& Raab-Traub, N. (2013). Epstein-Barr virus LMP1 modulates lipid raft microdomains and the vimentin cytoskeleton for signal transduction and transformation. Journal of Virology, 87(3), 1301-1311.

40. Xiao, L., Hu, Z. Y., Dong, X., Tan, Z., Li, W., Tang, M., et al. (2014). Targeting Epstein-Barr virus oncoprotein LMP1-mediated glycolysis sensitizes nasopharyngeal carcinoma to radiation therapy. Oncogene., 33(37), 4568-4578.

41. Lin, M. C., Lin, Y. C., Chen, S. T., Young, T. H., \& Lou, P. J. (2017). Therapeutic vaccine targeting Epstein-Barr virus latent protein, LMP1, suppresses LMP1-expressing tumor growth and metastasis in vivo. BMC Cancer, 17(1), 18.

42. Bhakat, K. K., Mokkapati, S. K., Boldogh, I., Hazra, T. K., \& Mitra, S. (2006). Acetylation of human 8-oxoguanine-DNA glycosylase by p300 and its role in 8-oxoguanine repair in vivo. Molecular and Cellular Biology, 26(5), 1654-1665.

43. Kohno, T., Shinmura, K., Tosaka, M., Tani, M., Kim, S. R., Sugimura, H., et al. (1998). Genetic polymorphisms and alternative splicing of the hOGG1 gene, that is involved in the repair of 8hydroxyguanine in damaged DNA. Oncogene., 16(25), 32193225.

44. Nishimura, S. (2002). Involvement of mammalian OGG1(MMH) in excision of the 8-hydroxyguanine residue in DNA. Free Radical Biology \& Medicine, 32(9), 813-821.

45. Galluzzi, L., Kepp, O., Tajeddine, N., \& Kroemer, G. (2008). Disruption of the hexokinase-VDAC complex for tumor therapy. Oncogene., 27(34), 4633-4635.

46. Yamaguchi, M., Kita, K., Miwa, H., Nishii, K., Oka, K., Ohno, T., et al. (1995). Frequent expression of P-glycoprotein/MDR1 by nasal T-cell lymphoma cells. Cancer., 76(11), 2351-2356.

47. Imai, K., Kamio, N., Cueno, M. E., Saito, Y., Inoue, H., Saito, I., et al. (2014). Role of the histone H3 lysine 9 methyltransferase Suv39 h1 in maintaining Epsteinn-Barr virus latency in B95-8 cells. FEBS Journal, 281(9), 2148-2158.

Publisher's note Springer Nature remains neutral with regard to jurisdictional claims in published maps and institutional affiliations. 